\title{
X-ray sources and magnetic reconnection in the X3.9 flare of 2003 November 3
}

\author{
A. M. Veronig ${ }^{1}$, M. Karlický ${ }^{2}$, B. Vršnak ${ }^{3}$, M. Temmer ${ }^{1}$, J. Magdalenić $^{3}$, B. R. Dennis ${ }^{4}$, W. Otruba ${ }^{5}$, and W. Pötzi ${ }^{5}$ \\ ${ }^{1}$ IGAM/Institute of Physics, University of Graz, Universitätsplatz 5, 8010 Graz, Austria \\ e-mail: asv@igam.uni-graz.at \\ 2 Ondřejov Observatory, Czech Academy of Sciences, Czech Republic \\ ${ }^{3}$ Hvar Observatory, Faculty of Geodesy, Kačićeva 26, 10000 Zagreb, Croatia \\ ${ }^{4}$ Laboratory for Astronomy and Solar Physics, NASA Goddard Space Flight Center, Greenbelt, MD 20771, USA \\ 5 Kanzelhöhe Solar Observatory, IGAM/Institute of Physics, University of Graz, 9521 Treffen, Austria
}

Received 22 March 2005 / Accepted 24 September 2005

\section{ABSTRACT}

Context. Recent RHESSI observations indicate an apparent altitude decrease of flare X-ray loop-top (LT) sources before changing to the commonly observed upward growth of the flare loop system.

Aims. We performed a detailed study of the LT altitude decrease for one well observed flare in order to find further hints on the physics of this phenomenon and how it is related to the magnetic reconnection process in solar flares.

Methods. RHESSI X-ray source motions in the 2003 November 3, X3.9 flare are studied together with complementary data from SXI, EIT, and Kanzelhöhe $\mathrm{H} \alpha$. We particularly concentrate on the apparent altitude decrease of the RHESSI X-ray LT source early in the flare and combine kinematical and X-ray spectral analysis. Furthermore, we present simulations from a magnetic collapsing trap model embedded in a standard 2-D magnetic reconnection model of solar flares.

Results. We find that at higher photon energies the LT source is located at higher altitudes and shows higher downward velocities than at lower energies. The mean downward velocities range from $14 \mathrm{~km} \mathrm{~s}^{-1}$ in the RHESSI 10-15 keV energy band to $45 \mathrm{~km} \mathrm{~s}^{-1}$ in the $25-30 \mathrm{keV}$ band. For this flare, the LT altitude decrease was also observed by the SXI instrument with a mean speed of $12 \mathrm{~km} \mathrm{~s}^{-1}$. RHESSI spectra indicate that during the time of LT altitude decrease the emission of the LT source is thermal bremsstrahlung from a "superhot" plasma with temperatures increasing from $35 \mathrm{MK}$ to $45 \mathrm{MK}$ and densities of the order of $10^{10} \mathrm{~cm}^{-3}$. The temperature does not significantly increase after this early (pre-impulsive superhot LT) phase, whereas the LT densities increase to a peak value of (3-4) $\times 10^{11} \mathrm{~cm}^{-3}$.

Conclusions. Modeling of a collapsing magnetic trap embedded in a standard 2D magnetic reconnection model can reproduce the key observational findings in case that the observed emission is thermal bremsstrahlung from the hot LT plasma. This agrees with the evaluated RHESSI spectra for this flare.

Key words. Sun: flares - Sun: X-rays, gamma rays

\section{Introduction}

The expansion of flare ribbons and the growth of the flare loop system in dynamical "two-ribbon" flares belong to the most convincing observational signatures of magnetic reconnection in the solar corona. Furthermore, observations of cuspedshaped structures in images from the Soft X-ray Telescope onboard Yohkoh (Tsuneta et al. 1992; Tsuneta 1996), the existence of hard X-ray (HXR) sources above the soft X-ray flare loops (Masuda et al. 1994), and upward ejected plasmoids (Shibata et al. 1995; Ohyama \& Shibata 1998) provide observational evidence that the energy release occurs above the flare loops.

In the classical "CSHKP" magnetic reconnection model of two-ribbon flares (Carmichael 1964; Sturrock 1966; Hirayama 1974; Kopp \& Pneuman 1976; for a review see Priest \& Forbes 2002), a sheared or twisted coronal arcade containing a prominence rises slowly during the preflare phase. Then the structure rapidly erupts and the arcade field lines are stretched to form a vertical current sheet above the magnetic inversion line. When the sheet gets long enough, fast reconnection starts (Furth et al. 1963; Ugai 1987; Vršnak et al. 2003; see also Török et al. 2004, and references therein). In this scenario, first the "inner" field lines come into contact, and the downward-shrinking reconnected field lines form the first (lowest lying) flare loops. In the course of time, the field lines rooted successively farther away from the inversion line reconnect. Consequently, the flare loop system grows and the flare ribbons move away from the inversion line. This is evident from many observations (Švestka et al. 1987; Doyle \& Widing 1990; Tsuneta et al. 1992; Švestka 1996; Sakao et al. 1998; Gallagher et al. 2002; Hudson \& Fletcher 2002; Krucker et al. 2003; Sui \& Holman 2003; Wang et al. 2003; Asai et al. 2004; Ji et al. 2004; Liu et al. 2004; Sui et al. 2004; Vršnak et al. 2004).

Here we present a multiwavelength analysis of the source motions in the X3.9 flare that occured on 2003 November 3 
with special emphasis on observations from the Reuven Ramaty High Energy Solar Spectroscopic Imager (RHESSI). The flare exhibits two strong footpoints and a distinct looptop (LT) source in X-rays as observed by the RHESSI instrument. A distinct post-flare LT source was observed by the Soft X-ray Imager (SXI) onboard the GOES-12 satellite, the Extreme-ultraviolet Imaging Telescope (EIT) onboard the Solar and Heliospheric Observatory (SoHO) and in $\mathrm{H} \alpha$ by the Kanzelhöhe Solar Observatory. These observations enable us to study the plasma evolution during the impulsive and late flare phases. In particular, high-quality observations of the flare loop system covering a broad spectral (temperature) range from $\mathrm{X}$-rays to $\mathrm{H} \alpha$ over a long time period provide detailed insight into the structure and evolution of the flare loop system (Lin 2004).

This paper is particularly inspired by recent findings from RHESSI observations of an apparent downward motion of the X-ray LT source during the very early phase of a flare before changing to the commonly observed upward expansion of the flare loop system (Sui \& Holman 2003; Krucker et al. 2003; Sui et al. 2004; Liu et al. 2004; Ji et al. 2004). Sui \& Holman (2003) and Sui et al. (2004) performed a detailed analysis of RHESSI observations of three homologous M-class flares that occurred during 2002 April 14-16. All three flares showed an apparent decrease of the LT altitude before and during the impulsive flare onset. The apparent loop height decrease was found to be more pronounced at higher X-ray energies. Furthermore, in all flares a source above the LT was observed during the time of the LT altitude decrease which was stationary for several minutes and eventually moved outwards at $\sim 300 \mathrm{~km} \mathrm{~s}^{-1}$ (see also Sui 2004).

These recent findings suggest that the altitude decrease of flare LT sources might be a common feature in the early phase of solar flares and is possibly closely related to the formation of a large-scale current sheet above the flare loops (Sui \& Holman 2003; Sui et al. 2004). In the 2003 November 3 flare, also an apparent LT altitude decrease before the onset of the impulsive HXR emission is observed as already reported in Liu et al. (2004). Here, we use a multiwavelength approach with emphasis on detailed RHESSI analysis in order to find further observational details on this intriguing phenomenon and to understand how it might relate to the overall flare/reconnection process. We also propose an interpretation of the observed LT altitude decrease in the frame of a collapsing magnetic trap model. The simulations show that the model can explain the main observational findings in case that the emission in the X-ray LT source is predominantly thermal bremsstrahlung from the hot flare plasma.

\section{Data}

Our main observational emphasis lies in X-ray observations from RHESSI (Lin et al. 2002), a NASA Small Explorer Mission launched on 2002 February 5, designed to study high energy solar flare emission from $3 \mathrm{keV}$ to $17 \mathrm{MeV}$, with high spectral and spatial resolution. The spectral resolution is $1 \mathrm{keV}$ in the range from 3 to several hundred $\mathrm{keV}$. The spatial resolution is as high as 2 '. 3 with a full-Sun field of view
(Lin et al. 2002). Throughout the flare, the RHESSI thick attenuators were in the field of view causing a rapid drop of the effective detector area at low photon energies (Smith et al. 2002). Thus, for the present study we did not consider RHESSI observations below $10 \mathrm{keV}$.

We analyze complementary $\mathrm{H} \alpha$ observations from the Kanzelhöhe Solar Observatory (KSO), Austria (Otruba 1999; Otruba \& Pötzi 2003). KSO routinely takes full-disk $\mathrm{H} \alpha$ images with a time cadence of $\sim 5 \mathrm{~s}$ and a spatial resolution of 2 '.2/pixel. When the flare-mode is triggered, additionally images in the blue and red wing of the $\mathrm{H} \alpha$ spectral line (at the off-band center wavelength of $\mathrm{H} \alpha-0.3 \AA$ and $+0.4 \AA$ ) are taken with a cadence of about 1 image per minute in each wing. Furthermore, the flare was recorded by the Soft X-ray Imager (SXI; Hill et al. 2005; Pizzo et al. 2005) onboard the GOES 12 spacecraft and in Fe XII $195 \AA$ by the Extreme-ultraviolet Imaging Telescope (EIT; Delaboudiniere 1995) onboard SoHO.

For one aspect of the study (comparing the LT evolution in different wavelengths), it was necessary to accurately co-align all image data sets. RHESSI has a very high precision pointing $\left(<1^{\prime \prime}\right)$ and roll angle information $(<1$ arcmin) which are achieved by the solar aspect system (SAS) and two redundant roll aspect systems (RAS and PMTRAS; cf. Hurford \& Curtis 2002; Fivian et al. 2002). Therefore, we co-aligned all other data sets with respect to the RHESSI images. The $\mathrm{H} \alpha$ flare images were co-aligned with the RHESSI flare images based on the two footpoints seen in both HXRs and $\mathrm{H} \alpha$ for different times of the flare evolution. We found that the $\mathrm{H} \alpha$ images were offset with respect to the RHESSI images by -2 " in $x$ and $+8^{\prime \prime}$ in $y$. After this correction, the $\mathrm{H} \alpha$ footpoints turned out to be also cospatial (to the accuracy of the spatial resolution of the EIT and $\mathrm{H} \alpha$ images of $\sim 2-3^{\prime \prime}$ ) with the footpoint regions inferred from EIT images. This implies that RHESSI and EIT are already correctly co-aligned. The SXI images were co-aligned comparing SXI and EIT pre-flare images of active regions with the result that SXI was offset with regard to EIT by $-16^{\prime \prime}$ in $x$ and $+4^{\prime \prime}$ in $y$. We stress that the uncertainties in the co-alignment are at least as high as the spatial resolution of the instruments; i.e. they are highest for SXI, which has a resolution of $5^{\prime \prime} /$ pixel.

\section{Observations and overview}

The X3.9 flare of 2003 November 3 took place in active region NOAA 10488 , one of the three large and flare-active regions (NOAA 10484, 10486, 10488) that produced the major flares during the October/November 2003 period of very high solar activity. NOAA 10488 was an extremely fast emerging and evolving active region. Figure 1 shows continuum images and longitudinal magnetic field maps of NOAA 10488 observed on 2003 October 31 and November 3 with the Michelson Doppler Imager (MDI; Scherrer et al. 1995) onboard SoHO. In total, NOAA 10488 produced 2 X-class flares, 7 M-class flares, and numerous smaller events. Both X-class flares occurred on 2003 November 3 (X2.7 flare, start: 01:10 UT; X3.9 flare, start: 09:40 UT), when the active region was close to the solar limb (cf. Fig. 1). 

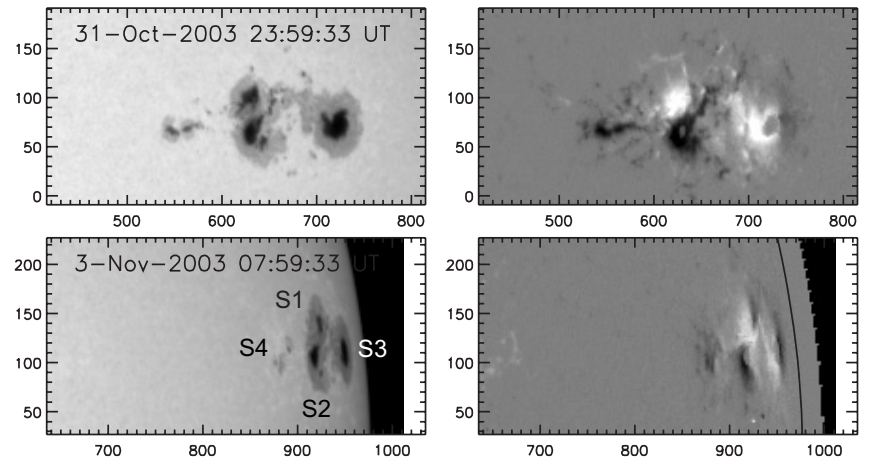

Fig. 1. NOAA 10488 as observed by SoHO/MDI on 2003 October 31 and November 3. The left panels show continuum images, the right panels show the corresponding longitudinal magnetograms (black: negative polarity, white: positive polarity). Each image is extracted from MDI full-disk observations and shows a $400^{\prime \prime} \times 200^{\prime \prime}$ field of view. Units of the $x$ - and $y$-axis are arcsec from disk center.

Figure $2 \mathrm{a}$ shows the GOES $0.5-4$ and $1-8 \AA$ time histories of the X3.9 flare on which we concentrate in this paper. A fast rise with a double peak is followed by a long decay phase of at least $5 \mathrm{~h}$. Figures $2 \mathrm{~b}-\mathrm{e}$ plot RHESSI light curves in 4 energy bands. Strong emission at high X-ray energies (cf. the $100-300 \mathrm{keV}$ energy band) is observed. The flare also produced $\gamma$-ray continuum emission (i.e. photon energies $\gtrsim 500 \mathrm{keV}$ ) but no $\gamma$-ray line emission was recorded by RHESSI. The flare basically consists of two main particle injection phases observed in HXRs (consistent with the double peak observed by GOES). Impulsive HXR emission with two major peaks is observed between $\sim 09: 48$ and 09:53 UT, followed by a comparatively "quiet" phase between 09:53 and 09:57 UT. A second impulsive phase starts at $\sim 09: 57$ UT and comprises five individual peaks up to the end of the RHESSI observations at 10:01:20 UT. When the RHESSI spacecraft went into the Earth shadow, there was still strong HXR emission present.

In Fig. 3 we show a sequence of RHESSI images reconstructed with the CLEAN algorithm (Hurford et al. 2002) using detectors 3 to 8 , which give an angular resolution of $\sim 7^{\prime \prime}$. The images obtained in the 15-20 keV energy band show a strong LT source which is present over the whole flare duration and can be imaged about 3-4 min prior to the impulsive HXR onset and footpoint appearance. The $70-100 \mathrm{keV}$ images show two distinct footpoints separating from each other during the course of the flare. The image sequence also indicates that the northern and southern footpoint alternate in which is stronger. Note that before 09:48:00 and from 09:54:10 to 09:56:40 UT it was not possible to reconstruct footpoint images; during these times the number of photons observed at high energies $(>50 \mathrm{keV})$ is very low (see Fig. 2).

Figure 4 shows a sequence of $\mathrm{H} \alpha$ images taken at KSO from the beginning to the late phase of the flare (from 09:44 to 12:31 UT). The images in panels (a) and (f)-(h) are taken in the center of the $\mathrm{H} \alpha$ spectral line, images (b)-(e) are taken in the red wing of the $\mathrm{H} \alpha$ spectral line $(+0.4 \AA)$. The line center images were almost immediately saturated after the impulsive flare onset. In contrast, the off-center images formed in deeper

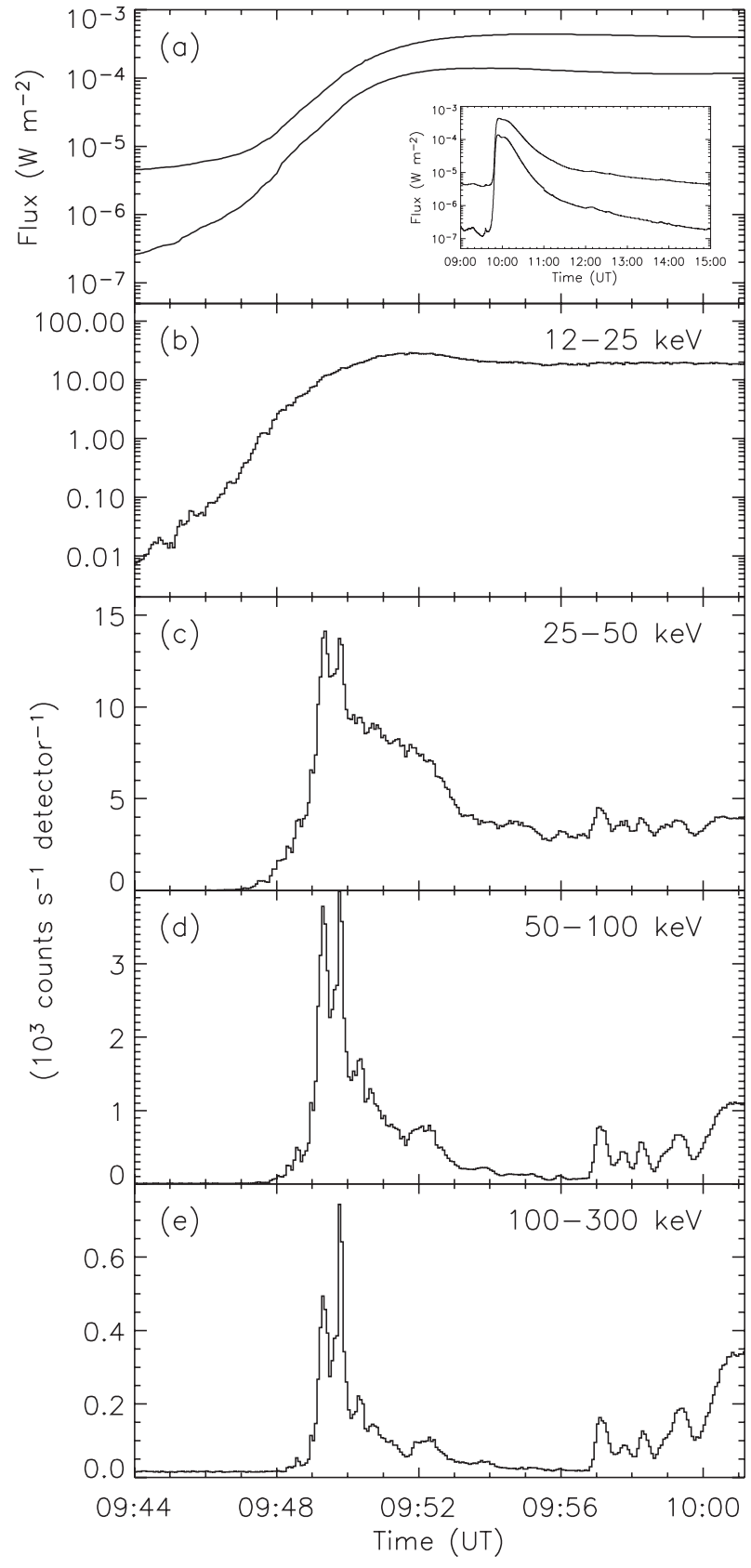

Fig. 2. GOES and RHESSI light curves for the impulsive phase of the 2003 November 3 X3.9 flare. a) GOES $0.5-4$ and 1-8 A fluxes. The insert plots the GOES evolution for the extended period 9:00 to 15:00 UT, i.e. showing also the rise and decay phase. b)-e) RHESSI light curves obtained in four energy bands with 4-s integration: 12-25, 25-50, 50-100 and 100-300 keV.

layers of the solar atmosphere which are not so strongly heated by the particle beams are less affected by saturation effects.

At the impulsive flare onset, three footpoints (each associated with one of the three large spots of the active region denoted as S1, S2 and S3 in Fig. 1) and a curved ribbon associated with S2 and the small preceding spot group S4 (both have negative polarity) are seen in $\mathrm{H} \alpha$. After about 09:55 UT only the two distinct footpoints associated with $\mathrm{S} 1$ (positive polarity) and S2 (negative polarity) are remaining. These footpoints coincide with the footpoints seen by RHESSI (cf. Figs. 3 and 4). 


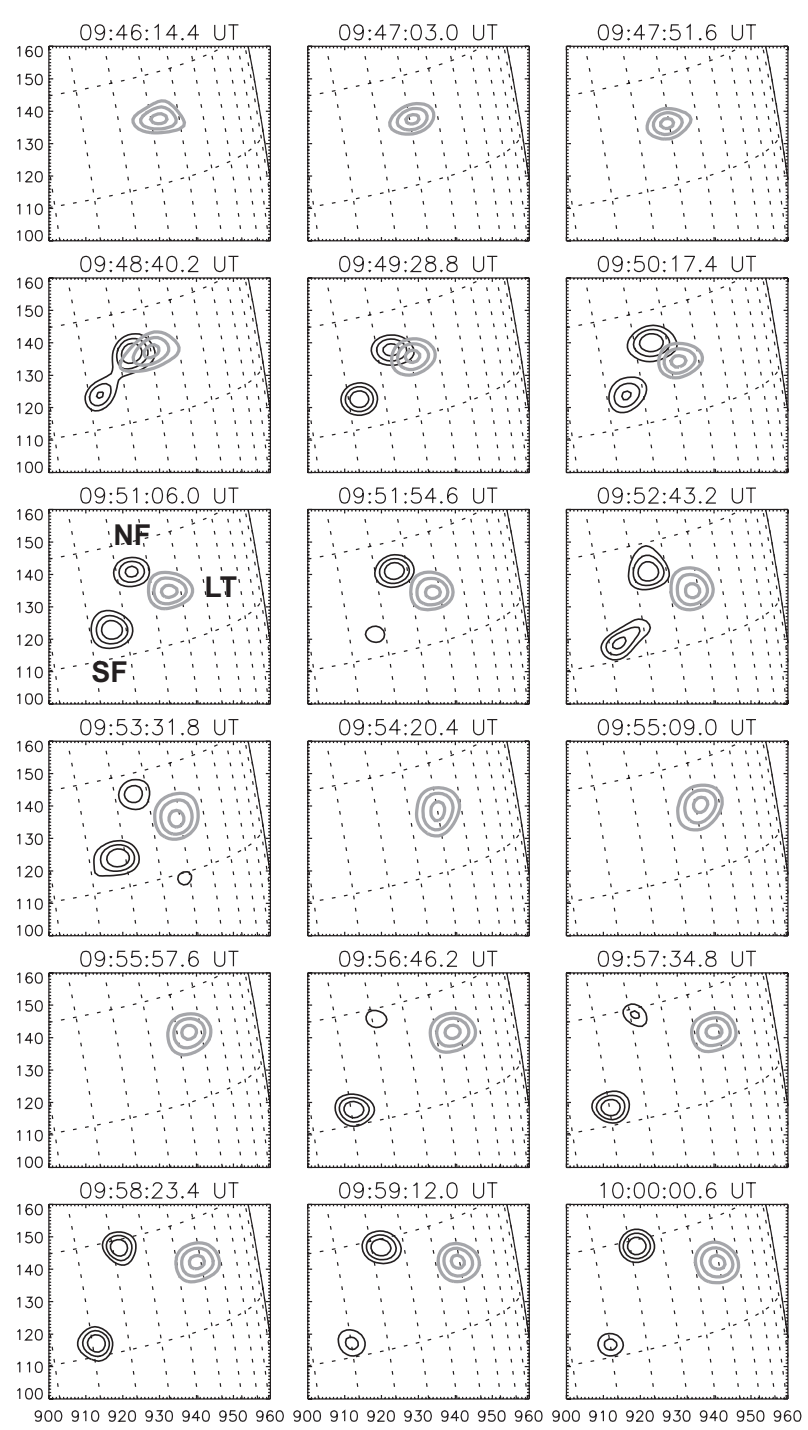

Fig. 3. Sequence of RHESSI 15-20 keV (gray contours) and 70-100 keV (black contours) images of the loop-top (LT) and footpoint (NF: Northern footpoint, SF: Southern footpoint) sources reconstructed with the CLEAN algorithm using grids 3 to 8 . For the 15-20 keV images the contour levels at 65,80 , and $95 \%$ of each image's peak flux are plotted. For the $70-100 \mathrm{keV}$ images the contour levels are 55,70 , and $85 \%$. The times given on each image refer to the start time of the 15-20 keV images (integration time for each image was $16.2 \mathrm{~s})$. The $70-100 \mathrm{keV}$ images are integrated over longer intervals (of individual length). The solar limb and a heliographic grid are also drawn in the images. Units of the $x$ - and $y$-axis are arcsec from disk center.

After 10:00 UT, in addition to the two footpoints a post-flare LT source is observed in the $\mathrm{H} \alpha$ images and can be followed for about three hours, first against the disk and later ( $\gtrsim 11: 30$ UT) above the solar limb.

Finally, we note that the event was associated with a fast CME $\left(\sim 1400 \mathrm{~km} \mathrm{~s}^{-1}\right)$, a diffuse Moreton wave, an EIT wave, a moving type IV burst and a complex type II burst extending to the hectometer wavelength range. Here we will not elaborate on these aspects; they are addressed elsewhere (Dauphin et al. 2005; Vršnak et al. 2005a,b).
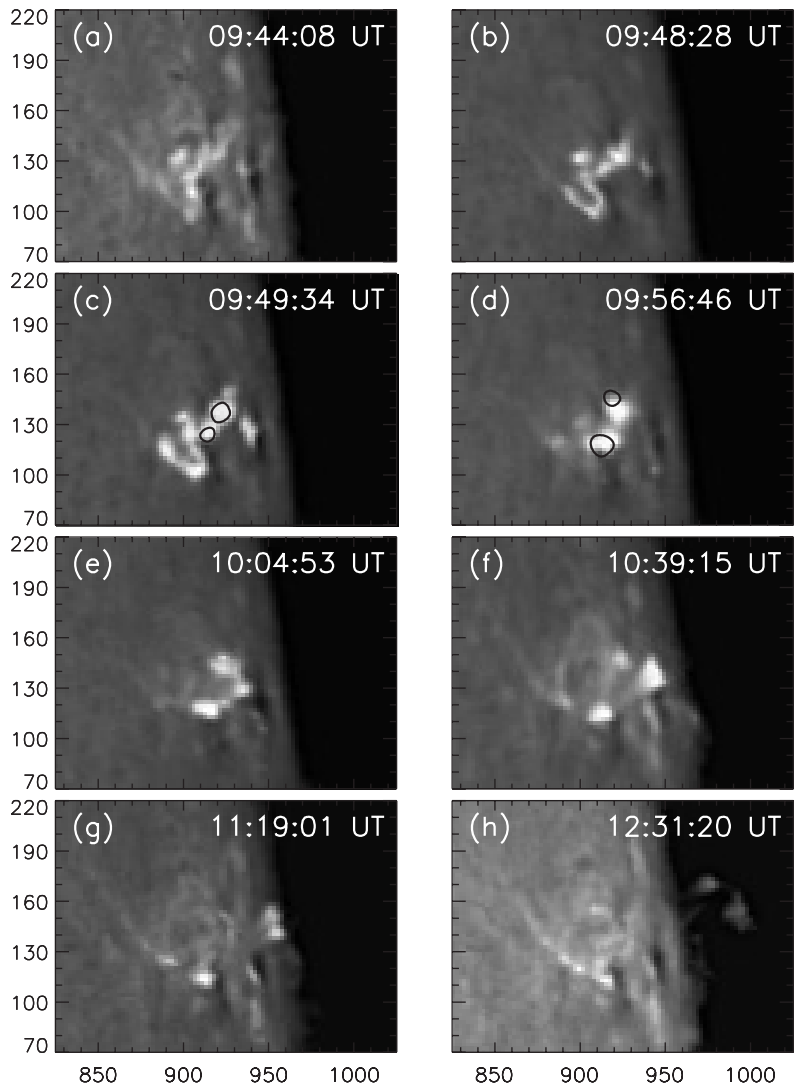

Fig. 4. Sequence of $\mathrm{H} \alpha$ images from the Kanzelhöhe Solar Observatory. The images in panels a) and f)-h) are taken in the center, images b)-e) in the red wing of the $\mathrm{H} \alpha$ spectral line. Each image is scaled separately. Units of the $x$ - and $y$-axis are arcsec from disk center. For comparison, in panels c) and d) contours of the RHESSI footpoints are drawn.

\section{Analysis and results}

\subsection{RHESSI LT and footpoint source motions}

In Fig. 5, the emission centroids of the RHESSI LT and footpoint sources are plotted on an MDI continuum image. All RHESSI images were derived with the CLEAN algorithm using front detector segments 3 to 8 . The images in the 10-15, 15-20, 20-25 and 25-30 keV bands were accumulated over consecutive $16.2 \mathrm{~s}^{\text {intervals }}{ }^{1}$. The $70-100 \mathrm{keV}$ images were

\footnotetext{
${ }^{1}$ We stress that in some intervals, in particular after about 09:49 UT when the count rates are highest and the detector livetimes drop sharply, the LT source can be also observed at energies $\gtrsim 30 \mathrm{keV}$. However, this source is most likely a "ghost" image caused by pulse pile-up whereby two (or more) low-energy photons arrive almost simultaneously at one detector and are recorded as a single photon with energy equal to the sum of the individual photon energies (see Smith et al. 2002; Hurford et al. 2002). With the present RHESSI software, pulse pile-up effects can be corrected in RHESSI spectra (this correction was used in the spectroscopy analysis in Sect. 4.2) but not in imaging. When the RHESSI thick attenuator is in, the peak counting rate is at $\sim 18 \mathrm{keV}$ and pulse pile-up is expected to be most severe at photon energies from about 30 to $50 \mathrm{keV}$ (though it may be important at even higher energies when more than 2 photons pile-up). Therefore, we did not consider LT images above $30 \mathrm{keV}$.
} 


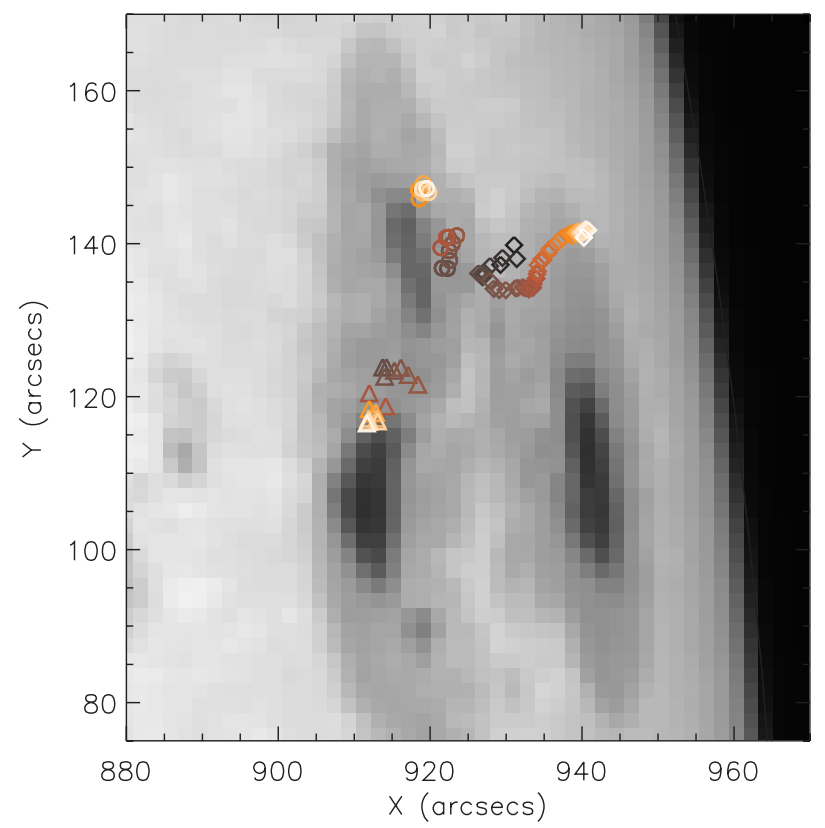

Fig. 5. Centroids of the RHESSI 15-20 keV LT (diamonds) and 70-100 keV footpoint (circles, triangles) sources on an MDI continuum image. The evolution in time is coded from black to white from 09:46:06-10:01:13 UT. The MDI image taken at 09:35:33 UT was differentially rotated to 10:00 UT.

integrated over longer intervals of individually chosen length to ensure sufficient count rates in each time bin. We tried different methods to determine the source locations: a) using a $3 \times$ 3 pixel parabolic fit around the brightest pixel; b) using the centroid of emission of all pixels above a certain threshold. We obtained the stablest results using the latter method with rather high thresholds. Finally, for the footpoint locations we used the centroid of emission of all pixels above $80 \%$ of the peak flux in the 70-100 keV images. For the LT source the centroid of emission of all pixels above $70 \%$ of the peak flux in the 10-15, 15-20, 20-25 and 25-30 keV images was used. In Fig. 5 the results for the $15-20 \mathrm{keV} \mathrm{LT}$ source and 70-100 keV footpoint sources are shown. Note that although the angular resolution in the reconstructed RHESSI images is $\sim 7^{\prime \prime}$, the emission centroids can be determined with an accuracy which may be $<1^{\prime \prime}$, depending on the count statistics (Hurford et al. 2002). To avoid errors in the source position which may occur when the count rate is low, we checked all reconstructed images together with the determined centroids. In particular, two images of the LT source sequences (around 09:49:00 UT) were rejected since at energies $\gtrsim 20 \mathrm{keV}$ the LT images were contaminated by emission from the Northern footpoint which was very close to the projected LT during this time (see Fig. 3).

Figure 5 illustrates that during the course of the flare the footpoints are separating from each other and the LT source appears to be progressing toward higher altitudes in the corona. (Note that the "jumps" in the footpoint positions between about 09:53 and 09:57 UT arise from the fact that during this time interval no accurate footpoint positions could be determined or no footpoints could be imaged at all.) During the very early phase, i.e. before the impulsive onset of

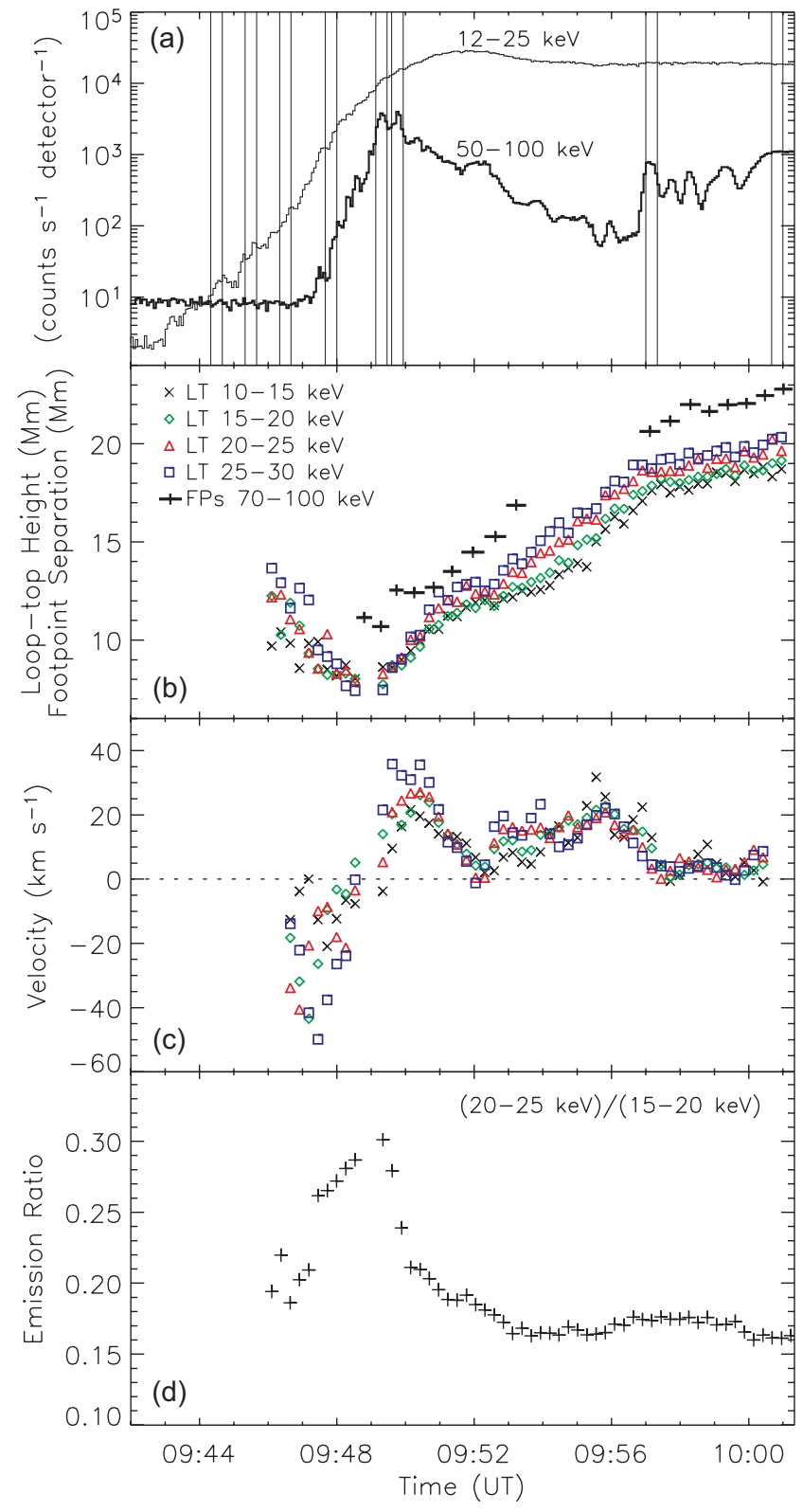

Fig. 6. a) Logarithmic plot of RHESSI 4-s light curves in the 12-25 keV and 50-100 keV energy bands (before background subtraction). The vertical lines indicate the accumulation times of the RHESSI spectra shown in Fig. 7. b) Evolution of the height of the LT source above the surface as derived from RHESSI images reconstructed in the 10-15, 15-20, 20-25, and 25-30 keV energy bands. Additionally, the footpoint separation derived from RHESSI 70-100 keV images is plotted. c) LT source velocity. d) Ratio of the LT source emission in the 20-25 and 15-20 keV bands.

HXR emission and before footpoints can be observed, the altitude of the LT source appears to be decreasing.

In Fig. 6b, the distance of the LT source along its main axis of motion for the 10-15, 15-20, 20-25 and 25-30 keV energy bands is plotted. The main axis of motion was determined by a linear least-squares fit to the LT centroid data and is offset from the radial direction by $18^{\circ}$ toward North. Also drawn is the separation of the two RHESSI footpoints reconstructed at 70-100 keV energies (not corrected for 
Table 1. Parameters of the LT altitude decrease as derived from linear fits to the RHESSI and SXI LT centroids. Initial and final LT altitudes refer to the times 09:45:58 and 09:48:24 UT.

\begin{tabular}{ccccc}
\hline \hline $\begin{array}{c}\text { Energy band } \\
(\mathrm{keV})\end{array}$ & $\begin{array}{c}\text { Initial LT altitude } \\
(\mathrm{Mm})\end{array}$ & $\begin{array}{c}\text { Final LT altitude } \\
(\mathrm{Mm})\end{array}$ & $\begin{array}{c}\text { LT altitude decrease } \\
(\%)\end{array}$ & $\begin{array}{c}\text { LT downward velocity } \\
\left(\mathrm{km} \mathrm{s}^{-1}\right)\end{array}$ \\
\hline RHESSI 25-30 & 13.8 & 7.3 & 47 & 45 \\
RHESSI 20-25 & 12.1 & 7.7 & 36 & 30 \\
RHESSI 15-20 & 11.7 & 7.5 & 36 & 29 \\
RHESSI 10-15 & 10.1 & 8.2 & 19 & 14 \\
\hline SXI & 8.6 & 7.0 & 20 & 12 \\
\hline
\end{tabular}

foreshortening effects as the event is very close to the limb and small errors are thus strongly enhanced by the correction). It can be seen that the footpoint separation and LT progression to greater heights in the corona show a similar evolution, and that the LT source reconstructed at higher energies are systematically located at higher altitudes. Such a behaviour is consistent with the CSHKP model, i.e., with the reconnection taking place in the vertical current sheet beneath the CME.

Figure $6 \mathrm{c}$ shows the velocity of the RHESSI LT source obtained as the time derivative of the LT centroid data in panel $b$. For the downward velocity, peak values up to $-50 \mathrm{~km} \mathrm{~s}^{-1}$ are reached. The highest upward velocities of the LT source (up to $35 \mathrm{~km} \mathrm{~s}^{-1}$ ) are observed close in time to the two highest HXR peaks of the first impulsive phase (cf. Fig. 6, panels a and c), which is consistent with the standard reconnection model. If the LT upward motion reflects progressive magnetic reconnection, then higher upward velocities are associated with higher reconnection rates (in case of a uniform magnetic field). On the other hand, higher reconnection rates are associated with more efficient particle acceleration and thus more HXR emission. However, note that during 09:54-09:57 UT where only weak HXR emission and no footpoints are observed, velocities in the range 15 to $20 \mathrm{~km} \mathrm{~s}^{-1}$ are obtained, whereas during the second impulsive phase with distinct HXR spikes comparatively low velocities of about $5 \mathrm{~km} \mathrm{~s}^{-1}$ are observed. Such a behaviour could be the result of an interplay between a variable reconnection rate and changing external Alfvén velocity (Lin 2002, 2004, and references therein) which can lead to a change of the relative importance of thermal/non-thermal (LT/FP) flare signatures (Vršnak \& Skender 2005).

RHESSI source motions of this flare were already studied by Liu et al. (2004). For the LT upward motion, we obtained partially different results than these authors. Liu et al. report mean upward velocities of only $4-5 \mathrm{~km} \mathrm{~s}^{-1}$ for the interval 09:48:10-09:49:50 UT and 15-21 $\mathrm{km} \mathrm{s}^{-1}$ for the interval 09:49:50-09:56:50 UT, respectively, and conclude that the LT upward motion is relatively slower during the more active phases of hard X-ray emission. This is in contrast to our results that the peak velocity of LT growth (up to $35 \mathrm{~km} \mathrm{~s}^{-1}$ during the interval $\sim 09: 49: 30-09: 50: 30$ UT) occurs close in time to the two strongest hard X-ray peaks. The main difference we can make out between their and our analysis is that Liu et al. didn't exclude LT images likely contaminated by emission from the Northern FP which was very close to the projected LT during 09:49:00 UT. This is probably the reason for the low velocities they derived for the 09:48:10-09:49:50 UT interval and could also explain the discrepancy between the high footpoint separation speed of $29 \mathrm{~km} \mathrm{~s}^{-1}$ and the low LT speed of 4-5 $\mathrm{km} \mathrm{s}^{-1}$ they report for the 09:48:10-09:49:50 UT period.

In this paper, our main interest lies in the apparent LT downward motion during the early flare phase. As an alternative velocity estimation, we applied linear fits to the LT centroids during the time of downward motion using 10 images reconstructed in each energy band during the period 09:45:58 to 09:48:24 UT. The fit results are summarized in Table 1 . We find a systematic increase in the mean downward velocity with energy: in the $10-15 \mathrm{keV}$ band it is $14 \mathrm{~km} \mathrm{~s}^{-1}$ whereas in the $25-30 \mathrm{keV}$ band the mean downward velocity is as high as $45 \mathrm{~km} \mathrm{~s}^{-1}$ (which corresponds to a decrease of the initial LT height of 47\%). This trend is in agreement with the downward velocities reported by Liu et al. (2004) for this flare. Furthermore, from the linear fits we obtain that the initial LT heights are systematically higher in higher energy bands (ranging from $10.1 \mathrm{Mm}$ in the $10-15 \mathrm{keV}$ band to $13.8 \mathrm{Mm}$ in the 25-30 keV band). At the end of the downward motion, the LT centroids in the different energy bands are all within $1 \mathrm{Mm}$ (between 7.3 and $8.2 \mathrm{Mm}$ ). Note that these phenomena can basically also be inferred from Fig. 6 (panels b and c) but are less obvious due to the scatter in the individual data points.

In Fig. 6d we plot the ratio of the LT emission in the 20-25 and the 15-20 keV bands. As can be seen, there is a distinct spectral change during the time where the decrease in the LT height is observed. Note that a similar behavior is obtained if we use the ratio of lower or higher energy bands where the LT source can be imaged, i.e. this spectral change is not an effect of the particularly chosen energy bands. If the emission in the LT source is the bremsstrahlung from an (iso-)thermal plasma, then the increase of this ratio would indicate that the temperature is increasing. On the other hand, if the LT emission is due to nonthermal bremsstrahlung then it would indicate that the injected electron spectrum is hardening. Each scenario as well as a combination of both, i.e. temperature increase and spectral hardening during the flare initial phase is physically plausible. Another interesting point is that the emission ratio peaks roughly at the time when the LT source motion changes from downward to the upward direction. In Sect. 4.2 we show RHESSI spectroscopy in order to obtain further information on the predominant emission process in the LT source. 

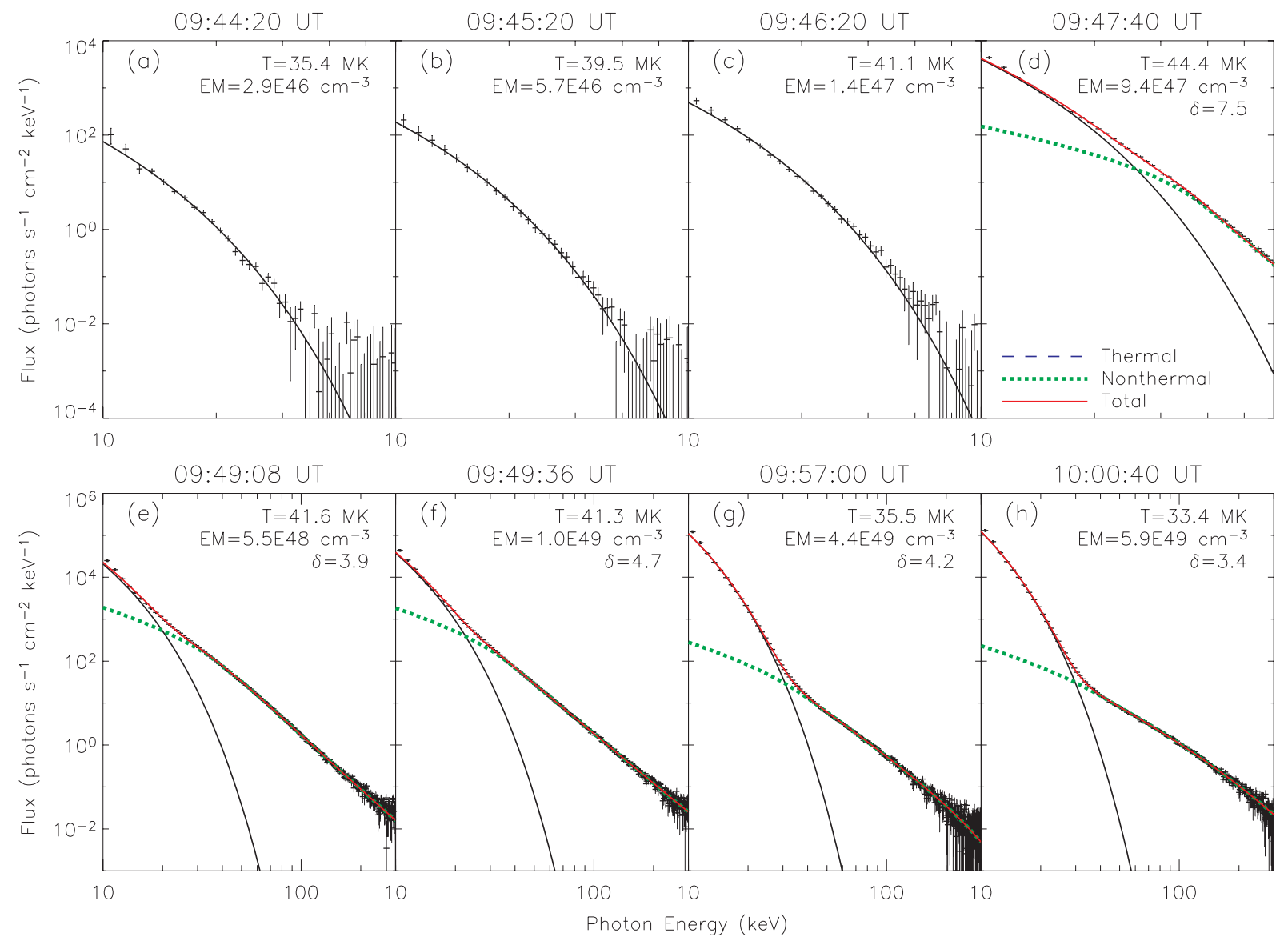

Fig. 7. RHESSI background-subtracted, spatially integrated photon spectra (crosses with error bars) derived during the very early flare phase (top panels) as well as during four main peaks at the impulsive phase (bottom panels). Each spectrum is accumulated over $20 \mathrm{~s}$ using all RHESSI front detector segments except 2, 5, and 7 (the start time of the integration is given in each panel; the accumulation periods are also indicated in Fig. 6a). Also shown are the model spectral fits: the dashed lines are for thermal bremsstrahlung; the dotted lines are for nonthermal thick-target bremsstrahlung with a low cutoff energy at $40 \mathrm{keV}$; the solid lines represent the sum of the two components. Temperature and emission measure of the thermal plasma as well as the (lower) spectral index $\delta$ of the accelerated electron spectrum according to the fits are given in each panel.

\subsection{RHESSI X-ray spectroscopy}

In Fig. 7 we show spatially integrated, background subtracted RHESSI spectra derived during eight time intervals of the flare together with the applied spectral fits. Our main emphasis lies on the very early phase, i.e. the time of altitude decrease of the LT source. The spectra were derived with $1-\mathrm{keV}$ bins accumulated over $20 \mathrm{~s}$ (the start time is indicated in each panel) using all front detector segments except 2, 5, and 7 (with lower spectral resolution and high threshold energies, respectively) and were corrected for pulse pile-up (Smith et al. 2002) using a "tweak" factor of 0.6.

In Fig. 6a, the 12-25 and 50-100 keV RHESSI light curves are plotted together with the accumulation times of the RHESSI spectra shown in Fig. 7. The plot reveals that the emission at low energies starts to gradually increase as early as 09:43:00 UT, whereas the increase of the 50-100 keV emission is not evident above the preflare background before 09:47:20 UT.

The spectra shown in Figs. 7a-d were derived during the very early flare phase (09:44:20-09:47:40 UT) where the emission is predominantly from the loop-top and no RHESSI footpoints can be imaged. For the spectra before 09:47:40 UT (Figs. 7a-c) we obtain acceptable fits, i.e. the reduced $\chi^{2} \sim 1$, with solely an isothermal component in the energy range 10-30 keV, whereas in all later spectra an additional power-law component was needed. The spectra in panels (d)-(h) were fitted with an isothermal component plus the thick-target bremsstrahlung emission from a (broken) powerlaw mean electron flux distribution with a low cutoff energy at $40 \mathrm{keV}$ (cf. Holman 2003). The fitting range was $10-80 \mathrm{keV}$ for the spectrum plotted in panel (d) and 10-220 keV for the spectra in panels (e)-(f). Assuming Poisson statistics plus a 5\% systematic uncertainty on every measured count rate, all fits shown in Fig. 7 gave an acceptable reduced $\chi^{2} \sim 1$, though we note that the fits are not unique (for more detailed discussions on this kind of forward fitting technique applied to RHESSI spectra see Holman 2003; Holman et al. 2003; Sui et al. 2005).

RHESSI provides us with the unique possibility to do imaging spectroscopy in X-rays (e.g. Krucker \& Lin 2002; Emslie et al. 2003). However, this is difficult to accomplish for the 
early phase of the 2003 November 3 flare. Firstly, because the count rates are low during this period; and secondly, the first time footpoints can be imaged, the Northern footpoint is close to the projected LT (see Fig. 3), and this makes it impossible to clearly separate the emission sources. However, based on the RHESSI imaging information, we can interpret the earliest spatially integrated spectra, i.e. panels (a)-(c), as spectra solely of the LT source, whereas spectra (e)-(h) are the combination of both LT and footpoint emission. Spectrum (d) is very probably also a combination of LT and footpoint spectra though it is not possible to image footpoints at this time (most probably due to the low number of counts at energies $\gtrsim 50 \mathrm{keV}$, see Fig. 6a).

We note that even at the very early phase of the flare, i.e. before the impulsive increase of emission at high energies, the RHESSI spectra are indicative of a "superhot" (Lin et al. 1981) plasma with temperatures increasing from $35 \mathrm{MK}$ at 09:44:20 UT to a peak value of 45 MK around 09:48:00 UT. At the very early stage between 09:44:20 and 09:46:20 UT, the emission measure derived from RHESSI thermal fits increases from $3 \times 10^{46}$ to $14 \times 10^{46} \mathrm{~cm}^{-3}$. During the main flare, the emission measure then increases by three orders of magnitudes up to a peak value of about $6 \times 10^{49} \mathrm{~cm}^{-3}$ at the end of RHESSI observations (10:01 UT), whereas the temperature does not further increase after 09:48 UT.

Some of the spectra shown in Figs. 7d-h were fitted with a single power-law whereas others yielded a better fit with a broken power-law mean electron flux distribution. In each panel, the electron power-law index $\delta$ derived from the fits is given (in case of a double power-law fit, the index derived at lower electron energies is shown). During the impulsive phase, the spectra are flat, being hardest at the end of RHESSI observations with $\delta=3.4$ (see Fig. 7h). In case of thick-target bremsstrahlung, the photon spectral index $\gamma$ is related to the electron spectral index $\delta$ by $\gamma=\delta-1$ (Brown 1971). Thus, the hardest photon spectral index during the flare is $\gamma=2.4$.

\subsection{Multiwavelength observations of the flare and post-flare LT source}

Figure 8 shows the evolution of the LT source during the impulsive and long decay phase of the flare as seen by different instruments. In addition to the RHESSI LT centroids, we measured by visual inspection the post-flare LT sources observed in soft X-rays by SXI, in Fe XII $195 \AA$ by EIT and in $\mathrm{H} \alpha$. The RHESSI instrument is particularly sensitive to flare plasmas in excess of $10 \mathrm{MK}$. SXI's maximum sensitivity is primarily in the temperature range $1-10 \mathrm{MK}$. For the LT kinematical measurements we used four different SXI analysis filters sensitive to different temperatures: open filter position (2.9-3.4 MK), the thin polyimide filter (3.8 MK), the medium polyimide filter (3.8-4.0 MK), and the thin beryllium filter (5.0 MK). Instrumental details and performance of SXI are described in Hill et al. (2005) and Pizzo et al. (2005). The bandpass of EIT Fe XII (195 $\AA$ ) is narrow with a peak formation temperature of 1.6 MK (Delaboudiniere 1995). However, the EIT $195 \AA$ band is also sensitive to $15-20$ MK plasma from
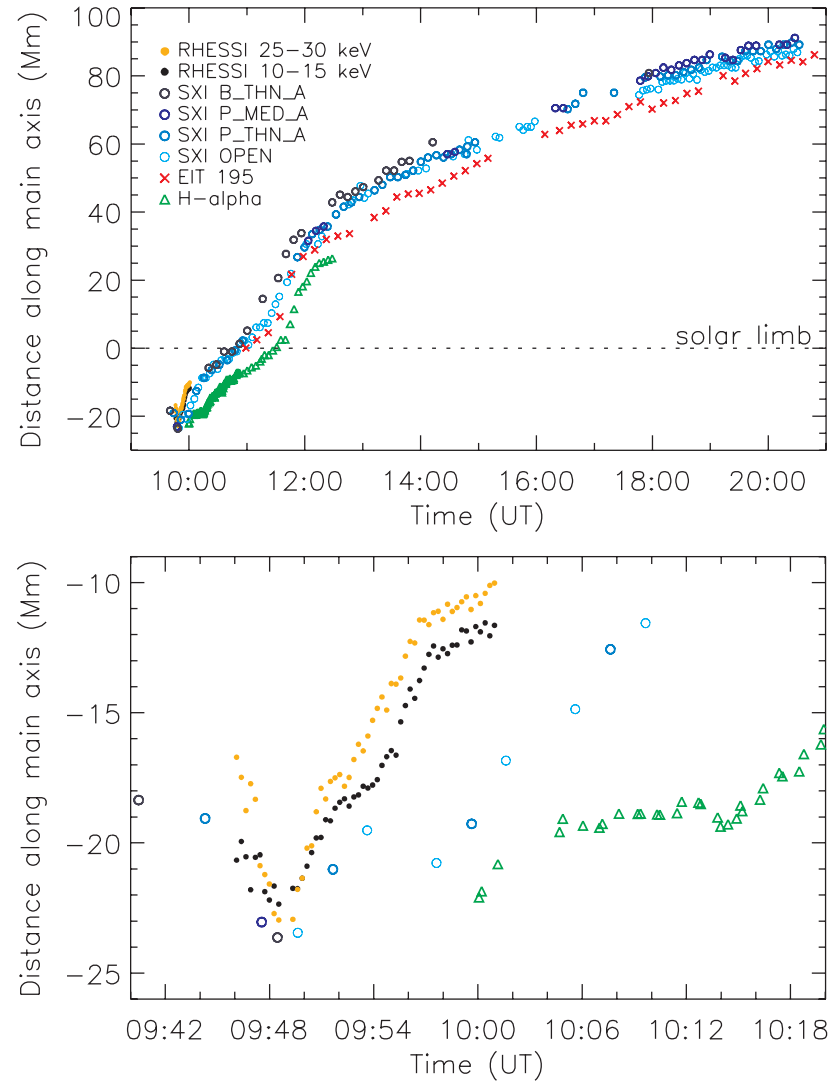

Fig. 8. Top: evolution of the flare and post-flare LT source as observed by RHESSI, SXI, EIT and KSO H $\alpha$. Bottom: zoom into the impulsive flare phase. The $y$-axis gives the distance measured along the main axis of the LT motion, which is interpreted as being source altitude (measured with respect to the solar limb).

the Fe XXII line. In $\mathrm{H} \alpha$ we see plasma at temperatures of about 10000 K (e.g. Švestka 1976; Heinzel \& Karlický 1987).

EIT observed an arcade of loops and several individual loops could be distinguished in $\mathrm{H} \alpha$. SXI only saw a bright blob probably because of the lower spatial resolution of $\sim 10^{\prime \prime} F W H M\left(5^{\prime \prime} /\right.$ pixel) compared to the EIT 2.'6/pixel and $\mathrm{H} \alpha 2$ '.2/pixel. From each of the image sequences of the different instruments, i.e. H $\alpha$, EIT and SXI, we derived the main direction of motion of the post-flare LT source by a linear fit to the centroid data resulting in a direction of motion which is $15-19^{\circ}$ North from the radial direction. This is consistent with the $18^{\circ}$ obtained from the RHESSI flare LT source. It is worth noting that in SXI and EIT the rising loop system could be observed up to the end of the day.

In Fig. 8 (top panel), the evolution is plotted up to about 21:00 UT, afterwards the features become too diffuse to obtain reasonable positional measurements. (Note that in EIT no measurements could be performed before 11:00 UT since the EIT detectors were saturated.) The figure reveals a clear structuring with temperature, i.e. higher temperature plasma is located above lower temperature plasma. From EIT and SXI measurements, we find for the late decay phase between about 14 and 21 UT an upward velocity which is still as high as $\sim 1.5 \mathrm{~km} \mathrm{~s}^{-1}$. 
The bottom panel of Fig. 8 zooms into the curve shown in the top panel, focusing on the flare initial and impulsive phase. It is interesting to note that SXI measurements also indicate an initial altitude decrease of the LT source before 09:49 UT. From a linear fit to the four earliest SXI data points which show the altitude decrease, we derive a mean downward velocity of $12 \mathrm{~km} \mathrm{~s}^{-1}$. This value is in agreement with the trend found from RHESSI observations that at lower photon energies the speed of LT altitude decrease is smaller (cf. Table 1).

The altitude measurements shown in Fig. 8 provide insight into the flare cooling process. In principle, at any given height the time difference between the measurements at different energies give an estimate of the time the flare plasma needs to cool (by conduction and radiation) from one temperature to the other. However, Švestka et al. (1987) noted that the loops also shrink while they cool by plotting the height of the hot SXR flare loops and the cool $\mathrm{H} \alpha$ post-flare loops as a function of time. As time progressed, the hot SXR loops (>10 MK) cooled to a temperature of the order of $10^{4} \mathrm{~K}$ where they became visible in the $\mathrm{H} \alpha$ spectral line. However, during the late phase of the flare the $\operatorname{cool} \mathrm{H} \alpha$ loops never reached the height of the hot SXR loops, and Švestka et al. (1987) concluded that the magnetic field lines on which the loops lie must have moved to lower altitudes as they cooled (so-called "loop shrinkage"; see also Forbes \& Acton 1996). Thus, the time delays between the loops observed at the same altitude but different temperatures represent upper limits to the plasma cooling times (see Švestka et al. 1987, Figs. 4 and 5 therein; Lin 2004, Figs. 5 and 6 therein).

From Fig. 8 we can infer that these upper limits for the cooling times increase with time. For instance, at 09:50 UT the hot flare LT plasma seen by RHESSI (35-45 MK) cools into the $\mathrm{H} \alpha$ passband $\left(10^{4} \mathrm{~K}\right)$ within $\lesssim 10 \mathrm{~min}$, at 09:54 UT the upper limit to the cooling time increased to $\sim 20-25 \mathrm{~min}$, and at 10:00 UT to $\sim 40-50 \mathrm{~min}$. Figure $8 \mathrm{~b}$ also shows that morphological structures in the LT altitude evolution (as, e.g., the "knee" in RHESSI measurements from 09:51 to 09:54 UT) are observed also in other wavelengths but delayed and prolonged in time. Such morphological features are important in order to disentangle the effects of flare loop cooling and shrinkage (Vršnak et al. 2005c).

\subsection{Density of the flare and post-flare LT source}

It is worth noting that only very rarely are $\mathrm{H} \alpha$ loops seen in emission against the disk and that such observations imply high electron densities in the post-flare $\mathrm{H} \alpha$ loops in excess of $10^{12} \mathrm{~cm}^{-3}$ as revealed from theoretical studies (Heinzel \& Karlický 1987; Švestka et al. 1987).

To obtain an estimate of the density $n$ of the hot flare plasma in the LT, we infer the source volume $V$ observed by RHESSI and determine emission measures $E M$ from RHESSI spectral fits, and calculated the density as $n=(E M / V)^{1 / 2}$ under the assumption of a filling factor of one. From RHESSI images reconstructed with the PIXON algorithm (Hurford et al. 2002) in the 15-20 keV band during the impulsive phase, we determined that the emitting area had a radial extent of about 15" (using the $50 \%$ contour levels in the PIXON images). Since the LT is the only emission source observed by RHESSI at these energies, we estimate the $\mathrm{X}$-ray emitting volume by approximating the LT source as a sphere with a diameter of $\sim 15^{\prime \prime}$ and obtain $V \approx 7 \times 10^{26} \mathrm{~cm}^{3}$.

From RHESSI spectral fits, we obtain a peak emission measure at the end of RHESSI observations of about $6 \times 10^{49} \mathrm{~cm}^{-3}$ which implies peak densities of the hot flare plasma as high as $3 \times 10^{11} \mathrm{~cm}^{-3}$. For the very early phase of the flare, i.e. during the time of LT altitude decrease, where the RHESSI emission measure increases from about $10^{46}$ to $10^{47} \mathrm{~cm}^{-3}$, we derive (assuming the same emitting source volume) plasma densities in the LT of the order of $10^{10} \mathrm{~cm}^{-3}$. At this period, the plasma temperature in the LT increases from about 35 to $45 \mathrm{MK}$.

For comparison, we derived also emission measure and temperature from GOES using the up-to-date Chianti atomic model (version 4.2; Young et al. 2003) recently implemented into the GOES analysis software (White et al. 2005). GOES observations reveal an enormous increase of the emission measure during the flare reaching its peak at 09:57 UT with a value of $1.4 \times 10^{50} \mathrm{~cm}^{-3}$. Using the same emitting source volume as observed by RHESSI, the GOES emission measures indicate peak densities as high as $4 \times 10^{11} \mathrm{~cm}^{-3}$. The peak temperatures derived from GOES at 09:51 UT is $26 \mathrm{MK}$ which is much smaller than that found from RHESSI spectral fits (45 MK). The result that RHESSI gives smaller emission measures and higher temperatures than GOES was found in several RHESSI studies and might be indicative of a multi-thermal plasma (e.g. Holman et al. 2003; Sui et al. 2005.) We stress that large uncertainties are involved in the flare density estimates. In particular, the volume estimates from the RHESSI images are quite uncertain and the filling factor may be as small as $10^{-4}$.

\subsection{RHESSI source above the $L T$}

Figure 9 shows a sequence of 15-20 keV RHESSI images derived during the flare rise phase. As can be seen from this sequence, a source above the LT develops and can be observed for about $100 \mathrm{~s}$ from 09:47:50 UT to 09:49:30 UT before it disappears from the images which is probably an effect of RHESSI's limited dynamic range ( 10:1; cf. Sui et al. 2004). As the reconstruction of this source is very much on the limits of RHESSI's imaging capabilities, we tested different image reconstruction algorithms (particularly CLEAN and PIXON), weighting schemes and energy bands. We note that the source can be seen with the different methods used at energies from about 10 to $30 \mathrm{keV}$. Thus, we are confident that the source above the LT is real. However, it is difficult to get insight into its evolution based, e.g., on the image sequence in Fig. 9, in particular whether it remains stationary over the period where it is observed or whether it is moving outwards.

The images shown in Fig. 9 were constructed with the CLEAN algorithm with natural weighting using RHESSI grids 3 to 8 . The source above the LT was best observed with natural weighting (see Fig. 9), most probably due to its better sensitivity for the detection of isolated compact sources, but it could also be seen in images reconstructed with uniform 


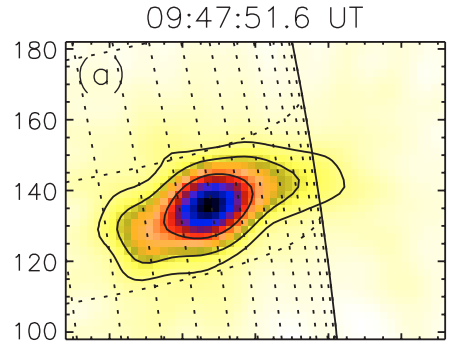

09:48:24.0 UT

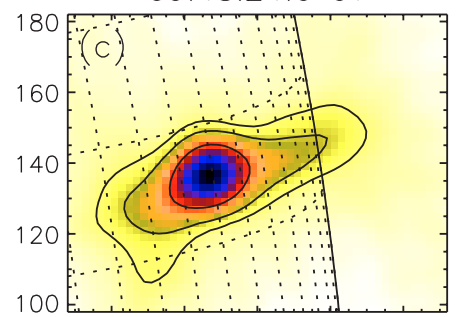

09:48:56.4 UT

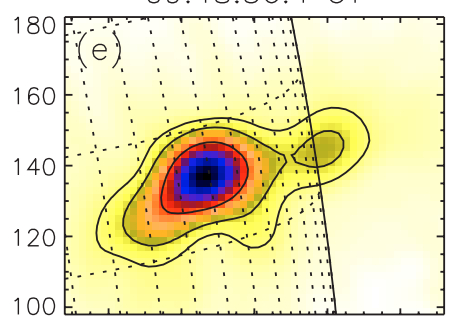

09:49:28.8 UT

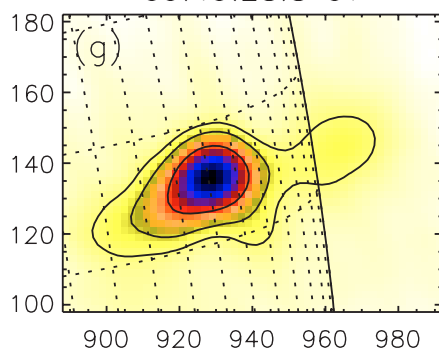

Fig. 9. Sequence of $15-20 \mathrm{keV}$ RHESSI images (for a $100^{\prime \prime} \times 80^{\prime \prime}$ field of view) showing the evolution of a source above the LT. Contour lines at 15, 25 and 50\% of each images's peak flux are plotted. The solar limb and a heliographic grid are also drawn in each panel.

weighting ${ }^{2}$. The line connecting the LT and the coronal source above the LT is offset from the radial direction by up to $\sim 10^{\circ}$.

\footnotetext{
${ }^{2}$ Natural weighting gives each of the chosen subcollimators equal weight, whereas in the uniform weighting scheme (which is the default in the RHESSI software) the finest subcollimators get much higher weight than the coarser ones. For RHESSI, uniform weighting assigns weights that are proportional to the subcollimator spatial frequency, viz. $1 /$ (spatial resolution). It follows that natural weighting has better sensitivity for the detection of isolated compact sources, and is also more sensitive to extended sources. However, due to the contribution of the coarser grids, the point response function has relatively strong "wings" extending out many beam widths from the core response. The angular resolution is also slightly degraded. Uniform weighting has a point response function that has slightly better angular resolution, no "wings" but larger sidelobes (Gordon Hurford, private communication).
}

Considering the measurement uncertainties due to the low image quality of the source above the LT, this value is similar to the $15-19^{\circ}$ derived for the main axis of motion of the flare and post-flare LT source.

Note that a source above the LT which was stationary for several minutes and eventually moved outwards was observed in all three flares studied by Sui \& Holman (2003) and Sui et al. (2004). Furthermore, in Sui \& Holman (2003) and Sui (2004) it was shown that in the LT source the temperature was increasing toward higher altitudes in the corona whereas in the coronal source above the LT it was decreasing toward higher altitudes. The authors concluded that the observed features are connected with the formation of a large scale current sheet between the LT and the coronal source above the LT.

\section{Interpretation of the LT altitude decrease}

Sui \& Holman (2003) and Sui et al. (2004) performed a systematic study of three homologous flares observed by RHESSI on 2002 April 14/15, 15, and 16. They found that in each of these flares the LT altitude decreased during the beginning of the flare rise phase, and only thereafter changed to the commonly observed upward motion. These authors report also a systematic difference of the LT height decrease observed for different energy bands in that higher energy sources were located higher in the corona and showed a faster altitude decrease than lower energy sources. In our case, it is not so obvious that during this time the emission centroids at higher energies are located at higher altitudes (cf. Fig. 6) but from the linear fits (Table 1) we also find that at higher X-ray energies the initial altitude of the LT source is higher and the downward velocities are larger than that at lower energies.

Sui et al. (2004) report speeds of the altitude decrease of $\sim 15-25 \mathrm{~km} \mathrm{~s}^{-1}$ in the 6-12 keV energy band, and $\sim 20-30 \mathrm{~km} \mathrm{~s}^{-1}$ in the $12-25 \mathrm{keV}$ band. These numbers are comparable to the velocities we derived for the 2003 November 3 flare (see Table 1). In our case, however, the LT source could be observed at even higher energies, and in the $25-30 \mathrm{keV}$ band we obtained a mean speed as high as $45 \mathrm{~km} \mathrm{~s}^{-1}$. Note that this velocity is higher than the largest upward velocity observed later in the flare $\left(\sim 35 \mathrm{~km} \mathrm{~s}^{-1}\right)$. We also stress that in this energy band, the decrease in LT altitude was almost $50 \%$ of the initial altitude (within a time range of 2-3 min).

One possible explanation of the observed LT altitude decrease is a geometrical effect caused, e.g., by the projection of successive brightenings along an arcade. However, an altitude change is suggested by the systematic findings that the phenomenon occurs only early in the flare, and that higher energies are located higher in the corona and reveal larger downward velocities (see also Sui et al. 2004).

Within the magnetic reconnection process in solar flares it is expected that newly reconnected field lines relax, "shrinking" down to form a system of closed loops (Švestka et al. 1987; Lin et al. 1995; Forbes \& Acton 1996; Lin 2004). Sui et al. (2004) suggested that the observed altitude decrease of the LT source might be also related to the change from slow $\mathrm{X}$-point to fast Petscheck-type reconnection which would not 
only increase the energy release rate but also push the lower bound of the current sheet downward.

In the following, we give an interpretation of the LT altitude decrease in the frame of a collapsing magnetic trap model (Somov \& Kosugi 1997; Karlický \& Kosugi 2004) embedded in a standard 2-D reconnection flare model, which is suitable for describing plasma processes in shrinking magnetic structures. In Sect. 5.1 we describe the model which is based on Karlický \& Kosugi (2004), and in Sect. 5.2 we present the simulation results. We show that the model can reproduce the key observational findings if we assume that the predominant emission process in the LT source is thermal bremsstrahlung.

For the 2003 November 3 flare, the LT X-ray source is observed in the energy range up to $\sim 30 \mathrm{keV}$. Therefore, the predominant emission mechanism in the LT source could be thermal bremsstrahlung of the hot flare plasma as well as nonthermal bremsstrahlung of electrons accelerated in the collapsing trap. (Though we note that the early RHESSI spectra favor a thermal origin, see Figs. 7a-c). In the following, we consider only thermal bremsstrahlung of the hot plasma in the collapsing magnetic trap. Namely, in the nonthermal model, accelerated electrons with higher energies penetrate deeper to lower altitudes of the solar atmosphere than do those with lower energies. During the time of altitude decrease, this would lead to a height structure in the LT source in which lower X-ray energies are located above higher X-ray energies. This is just the opposite of what is observed in this flare as well as those studied by Sui \& Holman (2003) and Sui et al. (2004). Therefore, in the modeling presented in the following we consider only thermal bremsstrahlung from the hot plasma in the collapsing magnetic trap.

\subsection{Collapsing magnetic trap: the model}

In the standard 2-D magnetic reconnection model of solar flares (e.g., Priest \& Forbes 2002) it is assumed that vertical, oppositely directed magnetic fields form a current sheet in which magnetic reconnection takes place and particles are accelerated. Acceleration in the DC electric field is often assumed to be the primary acceleration process (Litvinenko 1996). Among secondary acceleration processes there is one that naturally follows from the motion of the magnetic field lines in the cuspstructure below the current sheet and above the flare loops. In the reference system of the moving magnetic field lines and the flowing plasma, the magnetic field changes in time. This system of moving magnetic field lines was called the "collapsing magnetic trap" (Somov \& Kosugi 1997) and it was proposed to be an efficient particle accelerator, e.g. via the betatron mechanism.

In the betatron mechanism, particles are accelerated in the electric field $\boldsymbol{E}$ generated by time variations of the magnetic field $\boldsymbol{B}$ as it follows from Faraday's law $\nabla \times \boldsymbol{E}=-\partial \boldsymbol{B} / \partial t$. This type of acceleration was considered, for example, by Brown \& Hoyng (1975) and Emslie (1981) in connection with nonthermal and thermal HXR sources. However, not only suprathermal particles but also thermal particles suffer the influence of the betatron electric field $(\nabla \times \boldsymbol{E}=-\partial \boldsymbol{B} / \partial t)$. The betatron

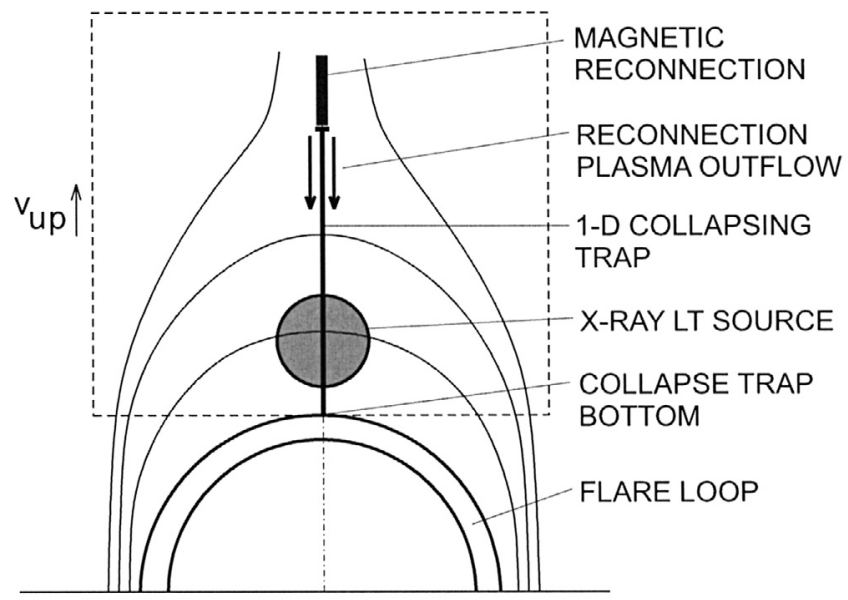

Fig. 10. Scheme of the X-ray LT source generated by accelerating and heating processes in the collapsing trap in the cusp magnetic field topology of flares. Due to successive loop formation under the trap, the magnetic field structure in the rectangular box as well as the 1-D collapsing trap moves upwards with a velocity $v_{\text {up }}$.

electric field always increases the momentum perpendicular to the magnetic field. For thermal particles, the temperature increases through an increase of the perpendicular temperature $T_{\perp}$ and collisional transfer to the parallel temperature $T_{\|}$. This process is called adiabatic heating (Emslie 1981) or betatron heating.

Figure 10 shows a sketch of the collapsing magnetic trap model embedded in a standard 2-D flare reconnection model. The plasma flowing from the reconnection site is injected into the collapsing trap (Karlický \& Kosugi 2004) and moves downwards together with magnetic field lines. At sites of increasing magnetic field strength the plasma is heated by the betatron heating mechanism. The process is most efficient in the central part of the collapsing trap structure, and hence this is where the $\mathrm{X}$-ray LT source is generated. Therefore, for the purpose of the present study, we consider only the processes along the vertical axis of the collapsing trap (1-D collapsing trap).

The flowing plasma stops at the position of the flare loop. However, due to successive loop formation, the stop position (the bottom of the collapsing trap) and the trap as a whole move upwards with a velocity $v_{\text {up }}$. (This process is similar to water falling into a pot with the consequent rise in the water level.) Assuming a stationary accumulation of magnetic field lines, the collapsing trap can be considered to be in a stationary state. In this case, the flow of the magnetic flux in the collapsing trap, in the coordinate system connected with this trap, is conserved:

$B v=C$

where $B$ is the magnetic field, $v$ the plasma velocity, and $C$ is a constant. To fit the plasma velocity at the bottom of the collapsing trap $v_{\text {bot }}$ with the upward velocity of the trap $v_{\text {up }}$ caused by successive loop formation, we put $v_{\text {bot }}=-v_{\text {up }}$. Namely, in the coordinate system fixed in the solar atmosphere, the plasma is stopped just at the bottom of the collapsing trap. On the other hand, the velocity of the plasma, flowing from the reconnection site and injected into the collapsing trap corresponds to 
the Alfvén speed of the plasma inflowing region (Priest 1982). Then, in the coordinate system connected with the trap, we can write

$B_{0} v_{0}=B_{\text {bot }} v_{\text {bot }}=B(h) v(h)$,

where $B_{0}$ and $v_{0}$ are the magnetic field and the plasma velocity in the collapsing trap near the reconnection site, respectively. $B_{\text {bot }}$ is the magnetic field at the collapse trap bottom, and $B(h)$ and $v(h)$ are the magnetic field and plasma velocity at an arbitrary height between the reconnection site and collapse trap bottom.

Now, let us specify the magnetic field and plasma velocity structure of the collapsing trap. In the stationary regime of the trap collapse, we define the time parameter $t_{h}$, which expresses the time that is needed to transport the plasma (and associated frozen-in magnetic field line) from the trap top to some specific height in the trap. Using this time parameter, we assume the magnetic field in the trap as

$B\left(t_{h}\right)=B_{0} \exp \left(t_{h} / t_{\text {char }}\right)$,

where $B_{0}$ is the magnetic field at the top of the trap, and $t_{\text {char }}$ is the characteristic time of the collapse.

Combining relations (1) and (3), we can write the expression for the plasma velocity in the collapsing trap as

$v\left(t_{h}\right)=v_{0} \exp \left(-t_{h} / t_{\text {char }}\right)$,

where $v_{0}$ is the plasma velocity at the top of the collapsing trap. Thus, the time when the flowing plasma reaches the collapse trap bottom is

$t_{h, \text { bot }}=-t_{\text {char }} \ln \left(\frac{v_{\text {bot }}}{v_{0}}\right)$.

Defining now the coordinate system $h_{\mathrm{b}}$ connected with the trap, for which at the collapse trap bottom $h_{\mathrm{b}}=0$, the height position of some specific magnetic field line for $t_{h}$ is

$h_{\mathrm{b}}=h-h_{0}$,

where we have $h=v_{0} t_{\text {char }} \exp \left(-t_{h} / t_{\text {char }}\right)$ and $h_{0}=$ $v_{0} t_{\text {char }} \exp \left(-t_{h, \text { bot }} / t_{\text {char }}\right)$.

Combining Eqs. (4) and (6), we find that in the stationary regime the relation between the height and the plasma velocity in the collapsing trap is

$h_{\mathrm{b}}=v t_{\mathrm{char}}-h_{0}$.

Knowing the height of some feature in the coordinate system connected with the trap, we can compute its height in the coordinate system fixed in the solar atmosphere as

$h_{\mathrm{a}}=h_{\mathrm{b}}+v_{\mathrm{up}} t+h_{00}$,

where $h_{00}$ is the height of the collapse trap bottom at the time $t=0$ in the coordinate system fixed in the solar atmosphere.

As concerns the density in the trap (in the coordinate system connected with the trap), we use the same parametric dependence as for the magnetic field

$n\left(t_{h}\right)=n_{0} \exp \left(t_{h} / t_{\text {char }}\right)$ where $n_{0}$ is the plasma density at the top position of the collapsing trap. Thus, the mass flow $n v$ in the collapsing trap in the coordinate system connected with the trap is conserved.

Now, knowing the magnetic field and density structure of the collapsing trap, we compute the evolution of the plasma temperature $T$ in the trap by specifying the plasma temperature $T_{0}$ at the injection position at the trap top at the initial time. For betatron heating in the collapsing trap, the temperature evolution is determined by (for details see Karlický \& Kosugi 2004)

$\frac{1}{T_{0}} \frac{\mathrm{d} T}{\mathrm{~d} t}=\frac{2}{3} \frac{1}{B_{0}} \frac{\mathrm{d} B}{\mathrm{~d} t}+H-Q$,

where $H$ is some additional heating, and $Q$ means energy losses (radiative and conduction losses). We can solve this equation numerically, or, if we assume $H-Q=0$ and the magnetic field according to Eq. (3), then the temperature in the trap is given as $T\left(t_{h}\right)=\frac{2}{3} T_{0} \exp \left(t_{h} / t_{\text {char }}\right)+\frac{1}{3} T_{0}$,

where $T_{0}=$ constant is the temperature of the plasma injected into the collapsing trap. The temperature profile according to Eq. (11) corresponds to the fully developed stationary regime of the collapsing trap. The maximum temperature is reached at the collapse trap bottom, which in the solar atmosphere moves upwards with the velocity $v_{\text {up }}$. However, at the beginning of flares the system is not immediately in the fully developed stationary regime but just evolves into it. Thus, for the demonstration of processes at the beginning of flares, we assume the magnetic field and plasma velocities in the trap are stationary, but we consider variations of the temperature of the plasma injected into the trap, i.e. $T_{0}(t)$ is a function of time. In this case the temperature in the collapsing trap can be expressed as

$T(h, t)=\frac{2}{3} T_{0}\left(t-t_{h}\right) \exp \left(t_{h} / t_{\text {char }}\right)+\frac{1}{3} T_{0}\left(t-t_{h}\right)$,

where $t_{h}=-t_{\text {char }} \ln \left[h /\left(v_{0} t_{\text {char }}\right)\right]$ is the time interval given by the plasma flow between the injection height and the specific height $h$.

Finally, the X-ray emission along the vertical axis of the trap is computed using the formula for thermal bremsstrahlung (Tandberg-Hanssen \& Emslie 1988):

$I\left(E_{x}\right)=D \frac{n^{2} V}{E_{x} T^{1 / 2}} \exp \left(-E_{x} / k T\right)$,

where $D$ is a constant, $E_{x}$ is the radiation energy, $T$ and $n$ are the plasma temperature and density, $V$ is the source volume, and $k$ is the Boltzmann constant. For more details about the model see Karlický et al. (2005).

\subsection{Model results}

For modeling, the following parameters of the collapsing trap were adopted: the plasma velocity, magnetic field and plasma density at the injection position in the trap are $v_{0}=$ $-800 \mathrm{~km} \mathrm{~s}^{-1}, B_{0}=5 \mathrm{G}$, and $n_{0}=5 \times 10^{9} \mathrm{~cm}^{-3}$. Then for the considered magnetic field at the region of the successive loop formation (the bottom of the collapsing trap) $B_{\text {bot }}=200 \mathrm{G}$, 


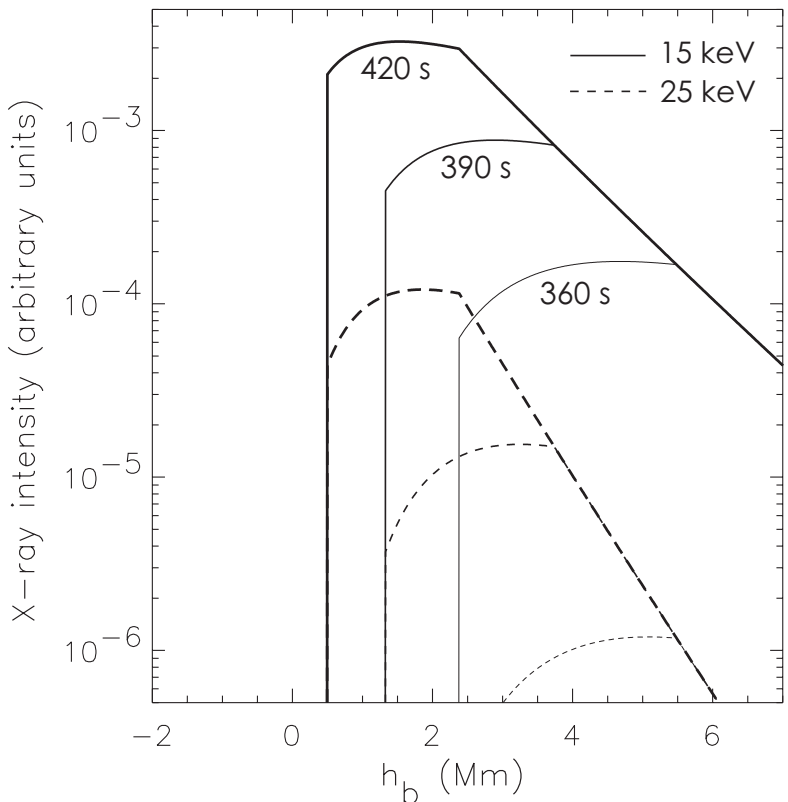

Fig. 11. X-ray intensity along the axis of the collapsing magnetic trap (in the coordinate system connected with the trap) at three instants: 360,390 , and $420 \mathrm{~s}$ (thin, thicker, and thickest lines, respectively) after the start of the plasma injection into the trap. $h_{\mathrm{b}}$ is the height in the collapsing trap ( $h_{\mathrm{b}}=0$ corresponds to the bottom of the collapsing trap). In the coordinate system fixed in the solar atmosphere the trap as a whole moves upward with a velocity $v_{\text {up }}=20 \mathrm{~km} \mathrm{~s}^{-1}$. Full lines correspond to a photon energy of $15 \mathrm{keV}$, dashed lines to $25 \mathrm{keV}$.

it gives an upward velocity of the growing loop system $v_{\text {up }}=$ $20 \mathrm{~km} \mathrm{~s}^{-1}=-v_{\text {bot }}$, and plasma density at the trap bottom as $n_{\text {bot }}=2 \times 10^{11} \mathrm{~cm}^{-3}$. For the characteristic time of the trap collapse, $t_{\text {char }}=120 \mathrm{~s}$ was chosen. We compare velocities of the observed X-ray sources with those in the bottom part of the collapsing trap. Thus, the considered characteristic time corresponds to the collapse process at this bottom part of the trap.

Initially, the temperature in the trap is assumed to be $T=$ $10^{6} \mathrm{~K}$ everywhere. At the injection position at the top of the trap, we considered a plasma with a temperature increasing in time as

$T_{0}(t)(\mathrm{K})=1.5 \times 10^{6}+2.5 \times 10^{4} t$, for $t \equiv\langle 0,60\rangle \mathrm{s}$,

$T_{0}(t)(\mathrm{K})=3 \times 10^{6}$ for $t \geq 60 \mathrm{~s}$.

We computed the time and spatial evolution of the temperature profile in the trap as well as the X-ray intensity profiles. The results of the computations at 360,390 , and $420 \mathrm{~s}$ after the first plasma injection into the trap are shown in Fig. 11. The maxima in both energy channels ( $15 \mathrm{keV}$ - full lines, $25 \mathrm{keV}$ dashed lines) move downwards to the trap bottom. Their velocities are slightly higher than the plasma velocities at these layers. When the plasma reaches the collapse trap bottom, its temperature rises to maximum values. Then the plasma escapes from the trap at its bottom boundary and is stopped in the solar atmosphere (due to the successive loop formation process). The betatron heating stops and the plasma starts to cool. The cooling process under the trap is not computed here. It is only assumed that there is a temperature decrease under the trap, and thus the temperature maximum remains at the bottom of

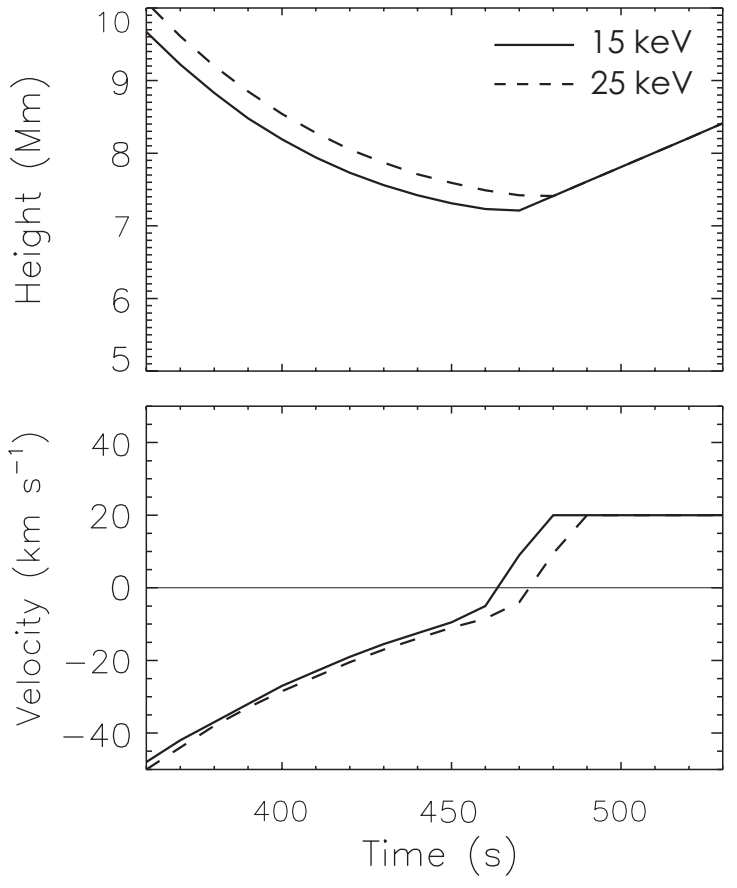

Fig. 12. Time evolution of the X-ray LT source altitude in the solar atmosphere (upper panel) and corresponding velocities (bottom panel). It was assumed that at the time $t=360 \mathrm{~s}$ the upward moving bottom of the collapse trap was located at a height of $5 \mathrm{Mm}$. Full lines represent the LT source at a photon energy of $15 \mathrm{keV}$, dashed lines are for $25 \mathrm{keV}$.

the collapsing trap which moves upward in the coordinate system fixed in the solar atmosphere. The maximum temperature in the trap reaches values from 40 to $80 \mathrm{MK}$.

Figure 12 shows the time evolution of the altitude (top panel) and velocity (bottom panel) of the X-ray LT source maxima for $15 \mathrm{keV}$ and $25 \mathrm{keV}$ photon energies in the coordinate system fixed in the solar atmosphere. Due to a superposition of the X-ray source motions in the trap and the upward motion of the trap itself, the downward motion of the X-ray sources eventually changes into an upward motion. The simulations show that the LT source at higher photon energy is located above that at lower energy and reveals larger downward velocities.

All these results were found in a simple model, neglecting, e.g., energy losses. These losses certainly modify the temperature profile of the flowing plasma in the trap and thus modify the distance between X-ray sources observed in different energy channels as well as their intensities.

\subsection{Model limitations and observational constraints}

We stress that our simple model is quite general in the sense that the magnetic collapsing trap process is always present in flares with a cusp-topology of magnetic field lines. However, there are also several limitations/assumptions involved which we will discuss in the following together with observational constraints.

The plasma velocity and magnetic field structure in the trap can, of course, be different in different flares. Besides the characteristic time of the collapse of the trap considered here and 
the subsequent betatron heating, other heating processes may be important such as irradiation, Alfvén waves, MHD shocks, etc. Also, both radiative and conductive energy loss processes must also be taken into account in any comprehensive model. For the present modeling, a relatively slow collapse process in the bottom part of the magnetic trap was considered, and it was assumed that the energy losses $Q$ are more or less compensated by additional heating $H$, i.e. $Q-H \approx 0$. When the betatron heating is smaller than $Q-H$, then we would not expect a LT source to be formed. Furthermore, if we increase the speed of the collapse process (i.e. shorten the characteristic time $t_{\text {char }}$ ), then the betatron heating can overcome losses but (considering the same increase of the magnetic field in the trap) the spatial extent of the trap would be smaller. This implies that a possible LT altitude decrease would occur over a smaller spatial scale and shorter time interval, and therefore be more difficult to observe.

We considered the collapsing trap with a stationary plasma flow. However, at the very beginning of the flare the stationary flow is not formed immediately but the system evolves into it. Nevertheless, we believe that also in this non-stationary case the physical processes in the collapsing trap will be similar. The plasma ejected from magnetic reconnection and injected into the trap will move downward and it will be heated in the compressed magnetic field up to the moment when the plasma flow stops at some altitude in the solar atmosphere and the accumulation of the magnetic field lines causes the upward motion of the whole structure. Further deviations from the stationary regime arise at times of variations of the reconnection rate, thus causing variations of the upward velocity $v_{\text {up }}$.

The LT altitude decrease early in the flare is a relatively new phenomenon revealed by RHESSI observations of at least five flares ${ }^{3}$. This is too few flares to allow any firm statistical conclusions to be drawn but in all cases the LT source was very strong with high plasma densities (peak values of a few times $10^{11} \mathrm{~cm}^{-3}$ ). The plasma density is a crucial parameter since the X-ray productivity depends strongly on density for both the thermal and nonthermal emissions. Thus, the observation of the phenomenon of the LT altitude decrease is restricted to LT sources which have a high enough density already at the very beginning of a flare. One possible scenario to provide enhanced plasma densities at the flare onset is consecutive flaring in the same set of loops in an active region. In this case a flare occurs in an already dense loop system filled by chromospheric evaporation during a preceding flare (such a scenario was, for instance, established for the 2002 April 14/15 flare by Veronig et al. 2005). We also note that for convincing observations of the LT altitude decrease phenomenon, a suitable "geometry"

32002 April 14/15 M3.7, April 15 M1.2, and April 16 M2.5 events cf. Sui \& Holman (2003), Sui et al. (2004) and Veronig \& Brown (2004). 2002 July 23 X4.8 flare cf. Holman et al. (2003), Lin et al. (2003) and Krucker et al. (2003). 2003 November 3 X3.9 flare see Liu et al. (2004) and the present paper. An altitude decrease of a RHESSI LT source is also mentioned for the M2.3 flare on 2002 September 9 by Ji et al. (2004) but no RHESSI images or LT measurements are presented in their paper. Furthermore, a RHESSI LT altitude decrease was also noticed for the X1.5 flare of 2002 April 21 (B. R. Dennis, unpublished). is required. In all the events where it was observed, the flare occurred close to the solar limb.

Finally, we stress that in a recent paper, Bogachev \& Somov (2005) studied the efficiency of betatron and first-order Fermi acceleration in collapsing magnetic traps. These authors find that depending on which process dominantes, there should exist two different types of coronal HXR sources: a) a high density source in case that the betatron mechanism is more efficient, and b) a source with lower density in case that the Fermi mechanism is more efficient. These findings are in agreement with our results obtained for the 2003 November 3 X3.9 flare showing a high density coronal source with a thermal bremstrahlung spectrum which could be modeled by betatron heating in a collapsing magnetic trap.

\section{Summary and conclusions}

We studied RHESSI X-ray sources in the 2003 November 3, X3.9 flare together with complementary data from SXI, EIT, and $\mathrm{KSO} \mathrm{H} \alpha$. Our main interest was in the structure and evolution of the flare loop system. Special attention was paid to the apparent altitude decrease of the RHESSI X-ray LT source early in the flare as recently discovered and studied in detail by Sui \& Holman (2003) and Sui et al. (2004) for three M-class flares on 2002 April 14 to 16. The key observational results agree with the findings reported by these authors: at higher photon energies the LT source is located at higher altitudes and shows higher downward velocities than at lower energies. In the 2003 November 3 flare, we observed mean downward velocities ranging from $14 \mathrm{~km} \mathrm{~s}^{-1}$ in the RHESSI $10-15 \mathrm{keV}$ energy band to $45 \mathrm{~km} \mathrm{~s}^{-1}$ in the $25-30 \mathrm{keV}$ band. We note that for this flare the LT altitude decrease was also observed by the SXI instrument. The derived speed was $12 \mathrm{~km} \mathrm{~s}^{-1}$, which is in agreement with the general trend that at lower photon energies the speed of LT altitude decrease is smaller.

From RHESSI spectral analysis we infer that during the time of LT altitude decrease, the emission of the LT source is most probably thermal bremsstrahlung from a "superhot" (Lin et al. 1981) plasma with temperatures increasing from $35 \mathrm{MK}$ to $45 \mathrm{MK}$ and densities of the order of $10^{10} \mathrm{~cm}^{-3}$. The temperature does not significantly increase after this early (preimpulsive) phase, whereas the LT densities increase to values as high as (3-4) $\times 10^{11} \mathrm{~cm}^{-3}$ before RHESSI observations were interrupted by the spacecraft's night time. During the impulsive rise phase, in addition to the LT and footpoint sources also an X-ray source above the LT was observed by RHESSI.

The pre-impulsive superhot coronal source is a very rare observational finding; to the awareness of the authors there is only one other paper (Uchida et al. 2001) where such a source was observed in Yohkoh data. However, theoretically it is not a difficulty to explain plasma temperatures of $45 \mathrm{MK}$ and higher during the flare initial phase by various mechanisms, e.g. by betatron (adiabatic) heating as studied in the present paper or by heating at the reconnection slow mode MHD shocks (e.g. Vršnak \& Skender 2005). From a $2 \frac{1}{2}$-D reconnection model, Vršnak \& Skender (2005) found that, at times of low reconnection rates (i.e. when the X-ray flux is relatively weak at the flare beginning and in the late phase), more particles stay trapped in 
the reconnection outflow region. This results in the absence of, or very weak, FP emission. Furthermore, they find that the thermal conduction flux becomes strongly inhibited leading possibly to the formation of a superhot LT source. The fact that such sources are only rarely observed may be an effect of low plasma densities and low emission measures, respectively.

The observational findings suggest that the observed LT altitude decrease is closely related to the initial phase of the magnetic reconnection process in solar flares. Within magnetic reconnection, several scenarios can possibly explain the observed LT altitude decrease: the relaxation of reconnected field lines, "shrinking" down to form a system of closed loops (Švestka et al. 1987; Lin et al. 1995; Forbes \& Acton 1996; Lin 2004); the change from slow X-point to fast Petscheck-type reconnection which would push downward the lower bound of the current sheet (Sui et al. 2004); plasma processes in shrinking magnetic structures as described in collapsing magnetic trap models.

Here, we have presented simulations from a magnetic collapsing trap model based on the model by Karlický \& Kosugi (2004). Though several simplifications were used, the model results are very promising. Not only do they reveal the general phenomenon of LT altitude decrease early in the flare but they also reproduce the systematically higher LT altitudes and downward velocities observed in higher energy bands. Note that this systematics is only reproduced by the model in case that the predominant emission process in the LT is thermal bremsstrahlung, whereas in the nonthermal case it is the opposite of the observed structuring. In the 2003 November 3 flare studied in this paper, the predominance of thermal bremsstrahlung emission in the LT is supported by the evaluated RHESSI spectra. We note, however, that for some flares evidence for nonthermal bremsstrahlung in flare LT sources has been presented (Sato 2001; Lin et al. 2003; Veronig \& Brown 2004).

Acknowledgements. We thank the RHESSI team for this highly successful mission and their open data policy. We also thank the SoHO and SXI teams for their open data policy. SoHO is a joint ESA/NASA mission. We thank the referee Richard Schwartz for insightful and detailed comments that helped to improve the paper. We also thank Gordon Hurford on discussions on RHESSI imaging issues and Linhui Sui for his comments on an earlier draft of this paper. This research was supported by Grant IAA3003202 of the Academy of Sciences, Grant 205/04/0358 of the Grant Agency of the Czech Republic and by the Austrian Science Fund (FWF project P15344).

\section{References}

Aschwanden, M. J., Schmahl, E. J., \& the RHESSI team 2002, Sol. Phys., 210, 193

Asai, A., Yokoyama, T., Shimojo, M., et al. 2004, ApJ, 611, 557

Bogachev, S. A., \& Somov, B. V. 2005, Astron. Lett., 31, 601

Brown, J. C. 1971, Sol. Phys., 18, 489

Brown, J. C., \& Hoyng, P. 1975, ApJ, 200, 737

Carmichael, H. 1964, in Physics of Solar Flares, ed. W. N. Hess, NASA SP-50, Washington, 451
Dauphin, C., Vilmer, N., Lüthi, T., et al. 2005, Adv. Space Res., 35, 1805

Delaboudiniere, J.-P., Artzner, G. E., Brunaud, J., et al. 1995, Sol. Phys., 162, 291

Doyle, J. G., \& Widing, K. G. 1995, ApJ, 352, 754

Emslie, G. A. 1981, ApJ, 244, 653

Emslie, A. G., Kontar, E. P., Krucker, S., \& Lin, R. P. 2003, ApJ, 505, L107

Fivian, M., Hemmeck, R., Mchedlishvili, A., \& Zehnder, A. 2002, Sol. Phys., 210, 87

Forbes, T. G., \& Acton, L. W. 1996, ApJ, 459, 330

Furth, H. P., Kileen, J., \& Rosenbluth, M. N. 1963, Phys. Fluids, 6, 459

Gallagher, P. T., Dennis, B. R., Krucker, S., Schwartz, R. A., \& Tolbert, A. K. 2002, Sol. Phys., 210, 341

Heinzel, P., \& Karlický, M. 1987, Sol. Phys., 110, 343

Hill, S. M., Pizzo, V. J., Balch, C. C., et al. 2005, Sol. Phys., 226, 255

Hirayama, T. 1974, Sol. Phys., 34, 323

Holman, G. D. 2003, ApJ, 586, 606

Holman, G. D., Sui, L., Schwartz, R. A., \& Emslie, G. A. 2003, ApJ, 595, L97

Hudson, H. S., \& Fletcher, L. 2002, Sol. Phys., 210, 307

Hurford, G. J., \& Curtis, D. W. 2002, Sol. Phys., 210, 101

Hurford, G. J., Schmahl, E. J., Schwartz, R. A., et al. 2002, Sol. Phys., 210,61

Innes, D. E., McKenzie, D. E., \& Wang, T. 2001, Sol. Phys., 217, 267

Ji, H., Wang, H., Goode, P. R., Jiang, Y., \& Yurchyshyn, V. 2004, ApJ, 607, L55

Karlický, M., \& Kosugi, T. 2004, A\&A, 419, 1159

Karlický, M., Veronig, A., \& Vršnak, B. 2005, Hvar Obs. Bull., in press

Kopp, R. A., \& Pneuman, G. W. 1976, Sol. Phys., 50, 85

Krucker, S., \& Lin, R. P. 2002, Sol. Phys., 210, 229

Krucker, S., Hurford, G. J., \& Lin, R. P. 2003, ApJ, 595, L103

Lin, J. 2002, Chinese J. Astron. Astrophys., 2, 539

Lin, J. 2004, Sol. Phys., 222, 115

Lin, J., Forbes, T. G., Priest, E. R., \& Bungey, T. N. 1995, Sol. Phys., 159,275

Lin, R. P., Schwartz, R. A., Pelling, R. M., \& Hurley, K. C. 1981, ApJ, 251, L109

Lin, R. P., Dennis, B. R., Hurford, G. J., et al. 2002, Sol. Phys., 210, 3

Lin, R. P., Krucker, S., Holman, G. D., et al. 2003, in Proceedings of the 28th International Cosmic Ray Conference, ed. T. Kajita et al. (Tokyo: University Academy Press), 3207

Litvinenko, J. E. 1996, ApJ, 462, 997

Liu, W., Jiang, Y. W., Liu, S., \& Petrosian, V. 2004, ApJ, 611, L43

Masuda, S., Kosugi, T., Hara, L., Tsuneta, S., \& Ogawara, Y. 1994, Nature, 371, 497

McKenzie, D. E., \& Hudson, H. S. 1999, ApJ, 519, L93

Ohyama, M., \& Shibata, K. 1998, ApJ, 499, 934

Otruba, W. 1999, in Third Advances in Solar Physics Euroconference: Magnetic Fields and Oscillations, ed. B. Schmieder, A. Hofmann, \& J. Staude, ASP Conf. Ser., 184, 314

Otruba, W., \& Pötzi, W. 2003, Hvar Obs. Bull., 27, 189

Pizzo, V. J., Hill, S. M., Balch, C. C., et al. 2005, Sol. Phys., 226, 283

Priest, E. R. 1982, Solar Magnetohydrodynamics (Dordrecht, The Netherlands: D. Reidel Publ. Comp.)

Priest, E. R., \& Forbes, T. G. 2002, Astron. Astrophys. Rev., 10, 313

Qiu, J., Lee, J., Gary, D. E., \& Wang, H. 2002, ApJ, 565, 1335

Sakao, T., Kosugi, T., \& Masuda, S. 1998, in Observational Plasma Astrophysics: Five Years of Yohkoh and Beyond, ed. T. Watanabe,

T. Kosugi, \& A. C. Sterling (Boston: Kluwer), 273

Sato, J. 2001, ApJ, 558, L137 
Scherrer, P. H., Bogart, R. S., Bush, R. I., et al. 1995, Sol. Phys., 162, 129

Shibata, K., Masuda, S., Shimojo, M., et al. 1995, ApJ, 451, L83

Smith, D. M., Lin, R. P., Turin, P., et al. 2002, Sol. Phys., 210, 33

Somov, B. V., \& Kosugi, T. 1997, ApJ, 485, 859

Sturrock, P. A. 1996, Nature, 211, 695

Sui, L. 2004, Modeling Solar Flare Hard X-ray Images and Spectra Observed with RHESSI, Ph.D. Thesis, Catholic University of America

Sui, L., \& Holman, G. D. 2003, ApJ, 596, L251

Sui, L., Holman, G. H., \& Dennis, B. R. 2004, ApJ, 612, 546

Sui, L., Holman, G. H., \& Dennis, B. R. 2005, ApJ, 626, 1102

Švestka, Z. F. 1976, Solar Flares (Dordrecht: Reidel)

Švestka, Z. F. 1996, Sol. Phys., 169, 403

Švestka, Z. F., Fontenla, J. M., Machado, M. E., Martin, S. F., \& Neidig, D. F. 1987, Sol. Phys., 108, 237

Tandberg-Hanssen, E., \& Emslie, A. G. 1988, The Physics of Solar Flares, Cambridge Astrophys. Ser. (Cambridge: Cambridge Univ. Press)

Török, T., Kliem, B., \& Titov, V. S. 2004, A\&A, 413, L27

Tsuneta, S., Hara, H., Shimizu, T., et al. 1992, PASJ, 44, L63
Tsuneta, S. 1996, ApJ, 456, 840

Ugai, M. 1987, Geophys. Res. Lett., 14, 103

Uchida, Y., Wheatland, M. S., Haga, R., Yoshitake, I., \& Melrose, D. 2001, Sol. Phys., 202, 117

Veronig, A. M., \& Brown, J. C. 2004, ApJ, 603, L117

Veronig, A. M., Brown, J. C., \& Bone, L. 2005, Adv. Space Res., 35, 1683

Vršnak, B., \& Skender, M. 2005, Sol. Phys., 226, 97

Vršnak, B., Klein, K.-L., Warmuth, A., Otruba, W., \& Skender, M. 2003, Sol. Phys. 214, 325

Vršnak, B., Maričić, D., Stanger, A. L., \& Veronig, A. 2004, Sol. Phys., 225, 355

Vršnak, B., Magdalenić, J., Temmer, M., et al. 2005a, ApJ, 625, L67

Vršnak, B., Warmuth, A., Temmer, M., et al. 2005b, A\&A, submitted

Vršnak, B., Veronig, A., Temmer, M., et al. 2005c, Sol. Phys., submitted

Wang, H., Qiu, J., Jing, J., \& Zhang, H. 2003, ApJ, 593, 564

White, S., Thomas, R. J., \& Schwartz, R. A. 2005, Sol. Phys., 227, 231

Young, P. R., Del Zanna, G., Landi, E., et al. 2003, ApJS, 144, 135 